\title{
The Hilbert null-cone on tuples of matrices and bilinear forms
}

\author{
Matthias Bürgin · Jan Draisma
}

Received: 8 August 2005 / Accepted: 6 January 2006 /

Published online: 28 June 2006

(C) Springer-Verlag 2006

\begin{abstract}
We describe the null-cone of the representation of $G$ on $M^{p}$, where either $G=\operatorname{SL}(W) \times \operatorname{SL}(V)$ and $M=\operatorname{Hom}(V, W)$ (linear maps), or $G=\operatorname{SL}(\mathrm{V})$ and $M$ is one of the representations $S^{2}\left(V^{*}\right)$ (symmetric bilinear forms), $\Lambda^{2}\left(V^{*}\right)$ (skew bilinear forms), or $V^{*} \otimes V^{*}$ (arbitrary bilinear forms). Here $V$ and $W$ are vector spaces over an algebraically closed field $K$ of characteristic zero and $M^{p}$ is the direct sum of $p$ of copies of $M$. More specifically, we explicitly determine the irreducible components of the null-cone on $M^{p}$. Results of Kraft and Wallach predict that their number stabilises at a certain value of $p$, and we determine this value. We also answer the question of when the null-cone in $M^{p}$ is defined by the polarisations of the invariants on $M$; typically, this is only the case if either $\operatorname{dim} V$ or $p$ is small. A fundamental tool in our proofs is the Hilbert-Mumford criterion for nilpotency (also known as unstability).
\end{abstract}

Keywords Representation theory $\cdot$ Null-cone $\cdot$ Matrices $\cdot$ Bilinear forms

Mathematics Subject Classification (2000) $\quad$ 14L24 · 20G05 $\cdot 15$ A63

\section{Introduction}

For a group $G$ and a finite-dimensional $G$-module $M$ over an algebraically closed field $K$, we denote by $K[M]^{G}$ the algebra of $G$-invariant polynomials on $M$. An element $m \in M$ is called nilpotent (or unstable) if it cannot be distinguished from 0 by $K[M]^{G}$, or, in other words, if all $G$-invariant polynomials on $M$ without constant term vanish on $m$. The nilpotent elements in $M$ form a (Zariski-)closed cone in $M$, called the

M. Bürgin $(\varangle)$

Mathematical Institute, University of Basel, Rheinsprung 21, 4051 Basel, Switzerland

e-mail: matthias.buergin@stud.unibas.ch

J. Draisma

Department of Mathematics and Computer Science, Technische Universiteit Eindhoven, P.O. Box 513, 5600 MB Eindhoven, The Netherlands

e-mail: jdraisma@win.tue.nl 
null-cone in $M$ ( $G$ being understood) and denoted $\mathcal{N}(M)=\mathcal{N}_{G}(M)$; it is a central object of study in representation theory. In this paper we will describe the irreducible components of the null-cone in some concrete representations.

We will, in fact, be studying the null-cone in a direct sum $M^{p}$ of $p$ copies of $M$, regarded as a $G$-module with the diagonal action. We recall some relations between the invariants and the null-cone of $M^{q}$ and those of $M^{p}$, where $p$ and $q$ are natural numbers. It is convenient, for this purpose, to identify $M^{p}$ with $K^{p} \otimes M$ where $G$ acts trivially on the first factor, and also, given a linear map $\pi: K^{p} \rightarrow K^{q}$, to use the same letter $\pi$ for the $G$-homomorphism $M^{p} \rightarrow M^{q}$ determined by $\pi(x \otimes m)=\pi(x) \otimes m, x \in K^{p}, m \in M$.

First, from an invariant $f \in K\left[M^{q}\right]^{G}$ we can construct $G$-invariants on $M^{p}$ as follows: for any linear map $\pi: K^{p} \rightarrow K^{q}$ the function $f \circ \pi$ is an invariant on $M^{p}$. The functions obtained in this way as $\pi$ varies are usually called polarisations of $f$ if $q \leq p$ and restitutions of $f$ if $q \geq p$. Using this construction, due to Weyl [18], it is easy to see that any linear map $\pi: K^{p} \rightarrow K^{q}$ maps $\mathcal{N}\left(M^{p}\right)$ into $\mathcal{N}\left(M^{q}\right)$ : indeed, an element $v$ of the former null-cone cannot be distinguished from 0 by any $G$-invariants on $M^{p}$, let alone by those of the form $f \circ \pi$ with $f \in K\left[M^{q}\right]^{G}$; hence $\pi(v) \in \mathcal{N}\left(M^{q}\right)$. Using this observation, we can prove that the number $c\left(M^{p}\right)$ of irreducible components of the $\mathcal{N}\left(M^{p}\right)$ behaves as follows.

Proposition 1 If $p \geq q$, then $c\left(M^{p}\right) \geq c\left(M^{q}\right)$. If in addition $q \geq \operatorname{dim} M$, then $c\left(M^{p}\right)=$ $c\left(M^{q}\right)$ and the polarisations to $M^{p}$ of the invariants on $M^{q}$ without constant term define the null-cone set-theoretically.

Proof Fix any surjective linear map $\pi: K^{p} \rightarrow K^{q}$; we claim that it maps $\mathcal{N}\left(M^{p}\right)$ surjectively onto $\mathcal{N}\left(M^{q}\right)$. Indeed, if $\sigma: K^{q} \rightarrow K^{p}$ is a right inverse of $\pi$, then any $v \in \mathcal{N}\left(M^{q}\right)$ is the image under $\pi$ of $\sigma v \in \mathcal{N}\left(M^{p}\right)$. This shows the first statement. For the second statement it suffices to prove that the map

$$
\phi: \operatorname{Hom}\left(K^{q}, K^{p}\right) \times \mathcal{N}\left(M^{q}\right) \rightarrow \mathcal{N}\left(M^{p}\right), \quad(\sigma, v) \mapsto \sigma v
$$

is surjective for $q \geq \operatorname{dim} M$, because the right-hand side has precisely $c\left(M^{q}\right)$ irreducible components. To prove surjectivity of $\phi$, let $v=\left(m_{1}, \ldots, m_{p}\right) \in \mathcal{N}\left(M^{p}\right)$. As $q \geq \operatorname{dim} M$, we can find a $w \in M^{q}$ whose components span the $K$-subspace $\left\langle m_{1}, \ldots, m_{p}\right\rangle_{K}$ in $M$. It follows that there exist linear maps $\pi: K^{p} \rightarrow K^{q}$ and $\sigma: K^{q} \rightarrow K^{p}$ such that $\pi v=w$ and $\sigma w=v$. We conclude that $w=\pi v$ lies in $\mathcal{N}\left(M^{q}\right)$ and $v=\phi(\sigma, w)$. The last statement is proved by a similar argument: suppose that all polarisations $f \circ \pi$ with $\pi \in \operatorname{Hom}\left(K^{p}, K^{q}\right)$ and $f \in K\left[M^{q}\right]^{G}$ without constant term vanish on $v \in M^{p}$, and let $h \in K\left[M^{p}\right]^{G}$ be without constant term. We can choose $\pi$ and $\sigma$ with $\sigma \pi v=v$ as before, and we find that $h(v)=((h \circ \sigma) \circ \pi) v=0$, because $(h \circ \sigma) \circ \pi$ is a polarisation of the $G$-invariant $h \circ \sigma$ on $M^{q}$.

Remark 1 In characteristic zero the last statement of Proposition 1 also follows from from Weyl's stronger result that the invariant ring on $M^{p}$ is generated by the polarisations of invariants on $M^{q}$ for $q \geq \operatorname{dim} V$ [18]. Weyl's theorem no longer holds in positive characteristic, though a weaker statement is still true [12]. However, an analogue of Weyl's theorem, for separating invariants, is true in arbitrary characteristic [6]-and, again, implies the last statement of Proposition 1.

Proposition 1 shows that $c\left(M^{p}\right)$ is an ascending function of $p$ that stabilises at some finite $p \leq \operatorname{dim} M$. This phenomenon was first observed by Kraft and Wallach in the case of reductive group representations [14], to which we turn our attention now. 
Suppose that $G$ is a connected, reductive affine algebraic group over $K$ and $M$ is a rational finite-dimensional $G$-module. One of the most important results on the nullcone in this setting is the Hilbert-Mumford criterion [15,16] for nilpotency: $v \in M$ lies in $\mathcal{N}(M)$ if and only if there exists a one-parameter subgroup $\lambda: K^{*} \rightarrow G$ such that $\lim _{t \rightarrow 0} \lambda(t) v=0$; we then say that $\lambda$ annihilates $v$. In this setting much more can be said about the irreducible components of the null-cone in $M^{p}$ : one verifies that for every one-parameter subgroup $\lambda$, the set

$$
G \cdot\left\{v \in M^{p} \mid \lim _{t \rightarrow 0} \lambda(t) v=0\right\}
$$

is a closed $G$-stable irreducible subset of $\mathcal{N}\left(M^{p}\right)$, and that a finite number of them cover $\mathcal{N}\left(M^{p}\right)$. Moreover, for $p$ sufficiently large, there are only the "obvious" inclusions among these sets [14] and this observations gives rise to a combinatorial algorithm for counting the irreducible components of $\mathcal{N}\left(M^{p}\right), p>>0$ [4]. However, for smaller values of $p$, there are usually many more inclusions, and our goal in this paper is to determine the exact "stabilising" value of $c\left(M^{p}\right)$ for the pairs $(G, M)$ in the abstract.

We note that the notion of "optimal" one-parameter subgroups for elements of the null-cone gives yet a finer description of the geometry of $\mathcal{N}(M)[11,16]$ - but this notion is not needed here.

Summarising, we will settle the following two fundamental problems for the pairs $(G, M)$ of the abstract: first, we describe the irreducible components of $\mathcal{N}\left(M^{p}\right)$ and determine at which value of $p$ their number stabilises; and second, we determine when $\mathcal{N}\left(M^{p}\right)$ is defined by the polarisations of the invariants on $M$. Note that in this case, by a result of Hilbert, the invariant ring of $M^{p}$ is finite over the subring generated by these polarisations [13, Section II.4.3]. The remainder of this paper has the following transparent organisation: Sections 2, 3, 4, and 5 deal with tuples of linear maps, symmetric bilinear forms, skew bilinear forms, and arbitrary bilinear forms, respectively. In the rest of the text we assume that $K$ has characteristic 0 ; this allows for the use of some "differential" arguments in the case of linear maps, while avoiding problems in small characteristics in the case of bilinear forms. However, most of what is proved here remains valid in arbitrary characteristic.

\section{Nilpotent tuples of linear maps}

For an $m$-dimensional vector space $V$ and an $n$-dimensional vector space $W$, both over our fixed algebraically closed field $K$ of characteristic 0 , the group $G=\operatorname{SL}(W) \times \operatorname{SL}(V)$ acts on the space $M=\operatorname{Hom}(V, W)$ of linear maps by $(g, h) A:=g A h^{-1}$. By duality we may assume that $0<m \leq n$, and we let $q:=\left\lceil\frac{n}{m}\right\rceil$ be the smallest integer $\geq n / m$. Then $\mathcal{N}\left(M^{p}\right)$ is as follows.

Theorem 1 The null-cone of $\operatorname{SL}(W) \times \operatorname{SL}(V)$ in $M^{p}=\operatorname{Hom}(V, W)^{p}$ consists of all p-tuples $\left(A_{1}, \ldots, A_{p}\right)$ of linear maps for which there exist subspaces $V^{\prime}$ of $V$ and $W^{\prime}$ of $W$ such that $n \cdot \operatorname{dim} V^{\prime}>m \cdot \operatorname{dim} W^{\prime}$ and $A_{i} V^{\prime} \subseteq W^{\prime}$ for all $i$.

The p-tuples for which $V^{\prime}$ can be chosen of a fixed dimension $k \in\{1, \ldots, m\}$ form a closed irreducible subset of $\mathcal{N}\left(M^{p}\right)$, denoted $C_{k}^{(p)}$. For $p<q$ the sets $C_{k}^{(p)}$ are all equal to $M^{p}$, and for $p>q$ they are precisely the distinct irreducible components of $\mathcal{N}\left(M^{p}\right)$. For $p=q$ there are still inclusions among the $C_{k}^{(q)}$, unless $m=1$, in which case $C_{1}^{(q)}=C_{1}^{(n)}=\mathcal{N}\left(M^{n}\right)$ is the irreducible null-cone consisting of singular $n \times$ 
$n$-matrices; or $n=(q-1) m+1$ with $q \geq 3$, in which case the $C_{k}^{(q)}$ are already the distinct components of the null-cone.

This theorem does not completely answer the question of how many irreducible components the null-cone on $q$ copies has. Some remarks on this matter can be found after the proof of the theorem, just before Example 2.

Somewhat prematurely, we will from now on call a pair $V^{\prime}, W^{\prime}$ as in the theorem a witness for the nilpotency of $\left(A_{1}, \ldots, A_{p}\right)$. In the proof that follows we use a theorem from elementary optimisation theory, the max-flow-min-cut theorem, which states that the maximal size of a flow from a source $s$ to a sink $t$ in a network equals the minimal capacity of a cut disconnecting $s$ from $t$. Here a network is a directed graph with two distinguished vertices $s$ and $t$ and a prescribed real-valued capacity function $c$ on the arrows; a flow is a real-valued function $f$ on the arrows that is bounded by $c$ and for which at every vertex other than $s$ and $t$ the sum of the $f$-values on the incoming arrows equals the sum of the $f$-values on the outgoing arrows; a cut is a set of arrows whose removal disconnects $s$ from $t$; and the capacities of a flow and of a cut are defined in the obvious manner. See [2, Chapter 3] for details.

Proof (Proof of Theorem 1, part one.) Suppose that $A=\left(A_{1}, \ldots, A_{p}\right)$ lies in the nullcone and let $(\mu, \lambda): K^{*} \rightarrow \mathrm{SL}(V) \times \mathrm{SL}(W)$ be a one-parameter subgroup annihilating $A$. Let $v_{1}, \ldots, v_{m}$ be a basis of $V$ with $\lambda(t) v_{j}=t^{a_{j}} v_{j}$, where $a_{j} \in \mathbb{Z}$, let $w_{1}, \ldots, w_{n}$ be a basis of $W$ with $\mu(t) w_{i}=t^{b_{i}} w_{i}$, where $b_{i} \in \mathbb{Z}$, and note that $\operatorname{det} \lambda(t)=\operatorname{det} \mu(t)=1$ implies $\sum_{j} a_{j}=\sum_{i} b_{i}=0$.

Now construct a directed graph $\Gamma$ with arrows of capacity $n$ from a source $s$ to $m$ vertices $1, \ldots, m$, arrows of capacity $m$ from $n$ vertices $\hat{1}, \ldots, \hat{n}$ to a sink $t$, and an arrow - for convenience, of infinite capacity - from $j$ to $\hat{\imath}$ if and only if $b_{i}-a_{j}>0$. See Fig. 1 for an example with $m=4$ and $n=6$. From

$$
\lim _{t \rightarrow 0} \mu(t) A_{k} \lambda(t)^{-1} v_{j}=\lim _{t \rightarrow 0} \mu(t) A_{k} t^{-a_{j}} v_{j}=0
$$

it is clear that each $A_{k}$ maps $v_{j}$ into the space spanned by the $w_{i}$ with $j \rightarrow \hat{i}$ in $\Gamma$. We claim that the maximal flow from $s$ to $t$ in $\Gamma$ is strictly smaller than the obvious upper

Fig. 1 The graph $\Gamma$ with a cut

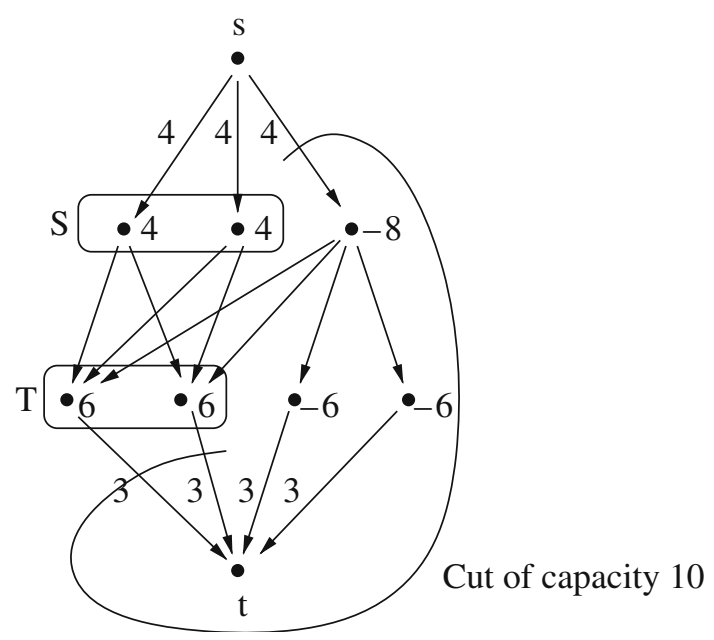


bound $m n$. Indeed, suppose that this upper bound were attained by a flow in which $c_{j, i}$ is the flow from $j$ to $\hat{i}$. Then $\sum_{i} c_{j, i}=n$ for all $j$ and $\sum_{j} c_{j, i}=m$ for all $i$, so that

$$
0=m \sum_{i} b_{i}-n \sum_{j} a_{j}=\sum_{j, i} c_{j, i}\left(b_{i}-a_{j}\right)
$$

but $c_{j, i}=0$ whenever $b_{i}-a_{j} \leq 0$, so that the right-hand side is strictly positive, a contradiction. Now the max-flow-min-cut theorem assures the existence of a cut of capacity strictly smaller than $m n$ and in particular not containing edges of infinite capacity. Let $T \subseteq\{\hat{1}, \ldots, \hat{n}\}$ be the set of vertices cut off from $t$, and let $S \subseteq\{1, \ldots, m\}$ be the set of vertices not cut off from $s$. By definition of a cut, no vertex $j$ of $S$ is connected to any vertex $\hat{\imath}$ outside of $T$, so that $V^{\prime}:=\left\langle v_{j} \mid j \in S\right\rangle_{K}$ is mapped by every $A_{k}$ into $W^{\prime}:=\left\langle w_{i} \mid \hat{\imath} \in T\right\rangle_{K}$. Finally, the capacity of the cut is equal to

$$
m|T|+n(m-|S|) \text { and by assumption }<m n,
$$

so that $m \operatorname{dim} W^{\prime}<n \operatorname{dim} V^{\prime}$ as required.

Conversely, suppose that $V^{\prime}, W^{\prime}$ is a witness for the nilpotency of $A$, set $(k, l):=$ $\left(\operatorname{dim} V^{\prime}, \operatorname{dim} W^{\prime}\right)$, and choose complements $V^{\prime \prime}$ and $W^{\prime \prime}$ of $V^{\prime}$ and $W^{\prime}$, respectively. Let $\lambda$ be the one-parameter subgroup of $\operatorname{SL}(V)$ having weights $a_{1}:=n(m-k)$ on $V^{\prime}$ and $a_{2}:=-n k$ on $V^{\prime \prime}$; note that $k a_{1}+(n-k) a_{2}=0$. Similarly, let $\mu$ be the one-parameter subgroup of $\operatorname{SL}(W)$ having weights $b_{1}:=m(n-l)$ on $W^{\prime}$ and $b_{2}:=-m l$ on $W^{\prime \prime}$. From the inequalities

$$
b_{1}-a_{1}>0, b_{1}-b_{2}>0, b_{2}-a_{1} \leq 0, \quad \text { and } \quad b_{2}-a_{2}>0
$$

we infer that $(\mu, \lambda)$ annihilates any linear map sending $V^{\prime}$ into $W^{\prime}$, so that $A \in \mathcal{N}\left(M^{p}\right)$. This proves the first statement of the theorem.

The sets $C_{k}^{(p)}$ from Theorem 1 are closed and irreducible by a general argument: they are of the form (1). Hence to prove the theorem we need only determine for what values of $p$ there are inclusions among the $C_{k}^{(p)}$. For this we need some auxiliary notation and results, which are of independent interest and which also give a formula for the dimensions of the irreducible components of $\mathcal{N}\left(M^{p}\right)$. We write $M_{a, b}$ for the space of $a \times b$-matrices with entries in $K$.

Definition 1 Let $a, b, c, d$, and $p$ be non-negative integers and let

$$
X_{i} \in M_{c, a} \text { and } Y_{i} \in M_{b, d} \quad \text { for } i=1, \ldots, p .
$$

Define the cut-and-paste map $\mathrm{CP}=\mathrm{CP}_{\left(X_{i}, Y_{i}\right)}: M_{a, b} \rightarrow M_{c, d}$ by

$$
\mathrm{CP} A=\sum_{i=1}^{p} X_{i} A Y_{i} \text {. }
$$

Now the rank of the linear map CP is clearly a lower semi-continuous function of the $p$-tuple $\left(X_{i}, Y_{i}\right)_{i}$, and we let $\mathrm{cp}^{(p)}(a, b, c, d)$, the cut-and-paste rank, be the maximal possible rank of CP, i.e., the rank for a generic $p$-tuple $\left(X_{i}, Y_{i}\right)_{i}$.

Remark 2 The following properties of the cut-and-paste rank are easy to check:

$$
\mathrm{cp}^{(p)}(c, d, a, b)=\mathrm{cp}^{(p)}(a, b, c, d)=\mathrm{cp}^{(p)}(b, a, d, c) .
$$


Indeed, the second equality comes from the fact that, upon composition with transposition on both sides, the cut-and-paste map $\mathrm{CP}_{\left(X_{i}, Y_{i}\right)_{i}}: M_{a, b} \rightarrow M_{c, d}$ yields $\mathrm{CP}_{\left(Y_{i}^{t}, X_{i}^{t}\right)_{i}}$ : $M_{b, a} \rightarrow M_{d, c}$; and the first equality reflects the fact that the transpose of $\mathrm{CP}_{\left(X_{i}, Y_{i}\right)_{i}}$ can be identified, via the trace form, with $\mathrm{CP}_{\left(X_{l}^{t}, Y_{i}^{t}\right)_{i}}: M_{c, d} \rightarrow M_{a, b}$. Moreover, if $a \leq c$ and $b \leq d$ then $\operatorname{cp}^{(p)}(a, b, c, d)=a b$ for all $p \geq 1$. Thus we reduce the computation of the cut-and-paste-rank to the case where $a b \leq c d, a \geq c$, and $b \leq d$. Then each of the maps $A \mapsto X_{i} A Y_{i}$ generically has rank $b c$, so that

$$
\mathrm{cp}^{(p)}(a, b, c, d) \leq \min \{a b, p b c\}
$$

Moreover, for $p \leq a / c$ it is easy to see that $\mathrm{cp}^{(p)}(a, b, c, d)$ is in fact equal to $p b c$ : by using suitable $X_{i}$ and $Y_{i}$, one can "cut" $p$ non-overlapping $c \times b$-blocks from an $a \times b$-matrix, and "paste" them in a non-overlapping way into a $c \times d$-matrix. The same argument shows that for $p$ sufficiently large $\mathrm{cp}^{(p)}(a, b, c, d)$ equals $a b$; this is the case, for example, as soon as one can cut an $a \times b$-matrix into $p$ non-overlapping rectangular blocks that fit without overlap into a $c \times d$-matrix. One might think that the inequality for the cut-and-paste-rank given above is always an equality, but this is not true: for $(a, b, c, d)=(5,4,3,7)$, for instance, we find cut-and-paste-ranks 12, 19, 20 for $p=1,2,3$, respectively. In short, we have no closed formula for $\mathrm{cp}$ and it would be interesting - but too much of a digression at this point in the paper-to find such a formula. In small concrete cases, however, the cut-and-paste rank can be computed easily; see below for some examples.

Proposition 2 Let $k, l, m, n, p$ be integers satisfying $0<k \leq m, 0 \leq l<n$, and $p \geq 0$. Then

$$
Q:=\left\{\left(A_{1}, \ldots, A_{p}\right) \in M_{n, m}^{p} \mid \exists U \subseteq K^{m}: \operatorname{dim} U=k \quad \text { and } \quad \operatorname{dim}\left(\sum_{i=1}^{p} A_{i} U\right) \leq l\right\}
$$

is an irreducible closed subvariety of $M_{n, m}^{p}$, and a sufficient condition for $Q$ to be strictly smaller than $M_{n, m}^{p}$ is

$$
p>\frac{l}{k}+\frac{m-k}{n-l} .
$$

Moreover, $\operatorname{dim} Q$ equals pmn if $p k \leq l$ and

$$
p m n-(p k-l)(n-l)+\mathrm{cp}^{(p)}(m-k, k, \min \{p(m-k), n-l\}, p k-l)
$$

otherwise.

Proof The set $Q$ is an irreducible closed variety because it is of the form (1), that is, the result of a vector space stable under a Borel subgroup of $G=\mathrm{SL}_{n} \times \mathrm{SL}_{m}$ being "smeared" around by $G$. For $p k \leq l$ the proposition is evident: any $p$-tuple maps any $k$-space into an $l$-space. Suppose therefore that $p k \geq l$. In the diagram

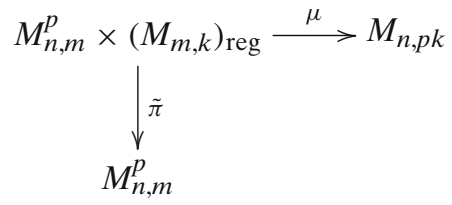


$\mu$ maps $\left(A_{1}, \ldots, A_{p}, B\right)$ to $\left(A_{1} B|\cdots| A_{p} B\right), \tilde{\pi}$ is the projection, and $\left(M_{n, k}\right)_{\text {reg }}$ is the set of rank $k$ matrices. Hence $Q=\tilde{\pi}\left(\mu^{-1}\left(X_{l}\right)\right)$, where $X_{l}$ is the variety of matrices in $M_{n, p k}^{p}$ having rank at most $l$. We will first compute the dimension of $Z:=\mu^{-1}\left(X_{l}\right)$ and then the dimension of a generic fibre of $\pi:=\left.\tilde{\pi}\right|_{Z}: Z \rightarrow Q$; the difference between these numbers is the dimension of $Q$.

First, $\mu$ is surjective and all its fibres have the same dimension $k m+p n(m-k)$. Indeed, for $\left(A_{1}, \ldots, A_{p}, B\right)$ to lie in the fibre over $\left(C_{1}, \ldots, C_{p}\right)$ we may choose $B \in$ $\left(M_{m, k}\right)_{\text {reg }}$ arbitrarily, and then each $A_{i}$ is determined on the $k$-dimensional image of $B$, but can still be freely prescribed on an $(n-k)$-dimensional complement. As $X_{l}$ has dimension $n l+p k l-l^{2}[10], Z$ has dimension $k m+p n(m-k)+n l+p k l-l^{2}$. Now $\mathrm{GL}_{k}$ acts freely on the fibres of $\pi$ by $g\left(\left(A_{i}\right)_{i}, B\right):=\left(\left(A_{i}\right)_{i}, B g^{-1}\right)$, so that

$$
\operatorname{dim} Q=\operatorname{dim} \pi(Z) \leq \operatorname{dim} Z-k^{2}=p n m-(p k(n-l)-k(m-k)-l(n-l)) .
$$

This implies the first statement of the proposition.

For the dimension of $Q$ we compute the dimension of a generic fibre $\pi^{-1} \pi(z)$ by computing the Zariski tangent space $T_{z} \pi^{-1} \pi(z)$, as follows. First, we show that $Z$ is irreducible and determine $T_{z} Z$ for generic $z \in Z$. Observe for this that the group $\mathrm{GL}_{m}$ acts on the fibres of $\mu$ by $g\left(\left(A_{i}\right)_{i}, B\right):=\left(\left(A_{i} g^{-1}\right)_{i}, g B\right)$. Now the map

$$
\begin{array}{r}
\phi: \mathrm{GL}_{m} \times M_{n, p k} \times M_{n, m-k}^{p} \rightarrow M_{n, k}^{p} \times M_{m, k}, \\
\left(g,\left(C_{1}|\cdots| C_{p}\right),\left(E_{i}\right)_{i}\right) \mapsto g\left(\left(C_{i} \mid E_{i}\right)_{i},\left(\frac{I_{k}}{0_{m-k, k}}\right)\right)
\end{array}
$$

maps $\mathrm{GL}_{m} \times X_{l} \times M_{n, m-k}^{p}$ surjectively onto $Z$, so $Z$ is irreducible as claimed. Furthermore, the map

$$
s: M_{n, m-k}^{p} \rightarrow M_{n, k}^{p} \times M_{m, k}, \quad x \mapsto \phi\left(1, x,(0)_{i}\right)
$$

is a right inverse of $\mu$, so by the chain rule $d_{z} \mu$ maps $M_{n, m}^{p} \times M_{m, k}$ surjectively onto $T_{\mu(z)} X_{l}$ for all $z \in M_{n, m}^{p} \times M_{m, k}$. In particular, if $z$ lies in $Z$ and $\mu(z)$ has rank exactly $l$ so that it is a smooth point of $X_{l}$, then we have

$$
T_{z} Z=\left(d_{z} \mu\right)^{-1} T_{\mu(z)} X_{l} .
$$

Now recall that if $\mu(z)$ has rank $l$, then

$$
T_{\mu(z)} X_{l}=\left\{N \in M_{n, p k} \mid N \operatorname{ker} \mu(z) \subseteq \operatorname{im} \mu(z)\right\} ;
$$

see [10, Example 14.16]. This will enable us to interpret the right-hand side in (2). On the other hand, because char $K=0$, we have

$$
T_{z} \pi^{-1} \pi(z)=\operatorname{ker}\left(d_{z} \pi: T_{z} Z \rightarrow T_{\pi(z)} Q\right)
$$

for generic $z \in Z$. Now let $z=\left(\left(A_{i}\right)_{i}, B\right) \in Z$ be generic. In particular, we require (2) and (4), and what further open conditions on $z$ are needed will become clear along the way. By the action of $\mathrm{GL}_{m}$ above we may assume that $B$ is of the form

$$
B=\left[\begin{array}{c}
I_{k} \\
0_{m-k, k}
\end{array}\right],
$$

and we split each $A_{i}=\left(A_{i, 1} \mid A_{i, 2}\right)$, accordingly. By genericity of the $A_{i}$ the matrix $\mu(z)=\left(A_{1,1}|\cdots| A_{p, 1}\right)$ has rank $l$, and by (2), (3), and (4) we find that $T_{z} \pi^{-1}(\pi(z))$ is isomorphic to the space of all $m \times k$-matrices 


$$
D=\left[\begin{array}{l}
D_{1} \\
D_{2}
\end{array}\right]
$$

such that

$$
\left(A_{1,1} D_{1}+A_{1,2} D_{2}|\ldots| A_{p, 1} D_{1}+A_{p, 2} D_{2}\right) \operatorname{ker} \mu(z) \subseteq \operatorname{im} \mu(z) .
$$

This is clearly the case for $D_{2}=0$ (this reflects the $\mathrm{GL}_{k}$-action used earlier), hence to determine what other $D$ have this property we may assume that $D_{1}=0$. The kernel of $\mu(z)$ has dimension $p k-l$, so we can choose $p$ matrices $Y_{1}, \ldots, Y_{p} \in M_{k, p k-l}$ such that the columns of the matrix

$$
\left[\begin{array}{c}
Y_{1} \\
\vdots \\
Y_{p}
\end{array}\right]
$$

form a basis of the kernel of $\mu(z)$. Again by genericity - the $A_{i, 2}$ are "independent" of the $A_{i, 1}$-the pre-image of $\operatorname{im} \mu(z)$ under $\left(A_{1,2}|\ldots| A_{p, 2}\right)$ has codimension $c:=$ $\min \{p(m-k), n-l\}$ in $K^{p(m-k)}$, and we may choose matrices $X_{1}, \ldots, X_{p} \in M_{c, m-k}$ such that the rows of $\left(X_{1}|\cdots| X_{p}\right)$ give linear equations for that inverse image. We now have

$$
\begin{aligned}
\left\{D_{2}\right. & \left.\in M_{m-k, k} \mid\left(A_{1,2} D_{2}|\cdots| A_{p, 2} D_{2}\right) \operatorname{ker}\left(A_{1,1}|\cdots| A_{p, 1}\right) \subseteq \operatorname{im}\left(A_{1,1}|\cdots| A_{p, 1}\right)\right\} \\
& =\left\{D_{2} \in M_{m-k, k} \mid \sum_{i} X_{i} D_{2} Y_{i}=0\right\} \\
& =\operatorname{ker}\left(\mathrm{CP}_{\left(X_{i}, Y_{i}\right)_{i}}: M_{m-k, k} \rightarrow M_{c, p k-l}\right) .
\end{aligned}
$$

Finally, because the $X_{i}$ and $Y_{i}$ are generic along with the $A_{i}$, the dimension of this space is $(m-k) k-\mathrm{cp}^{(p)}(m-k, k, c, p k-l)$. The dimension of the fibre $\pi^{-1}(\pi(z))$ is therefore $k^{2}$ plus this number, and we find

$$
\begin{aligned}
\operatorname{dim} \pi(Z)= & \operatorname{dim} Z-\operatorname{dim} \pi^{-1} \pi(z) \\
= & k m+p n(m-k)+n l+p k l-l^{2} \\
& -\left(k^{2}+(m-k) k-\mathrm{cp}^{(p)}(m-k, k, \min \{p(m-k), n-l\}, p k-l)\right) \\
= & p m n-(p k-l)(n-l) \\
& +\operatorname{cp}^{(p)}(m-k, k, \min \{p(m-k), n-l\}, p k-l),
\end{aligned}
$$

as claimed.

Remark 3 The difference $\operatorname{dim} \pi^{-1}(\pi(z))-k^{2}$, expressed above as the nullity of a certain cut-and-paste map, is the dimension of the variety of $k$-dimensional subspaces $U$ for which $\sum_{i} A_{i} U$ is at most $l$-dimensional.

Example 1 Proposition 2 is particularly useful to prove the existence of tuples of matrices not mapping any subspace of dimension $k$ into a subspace of dimension $l$. Consider the following two questions.

1. Do all triples $\left(A_{1}, A_{2}, A_{3}\right)$ of $8 \times 5$-matrices map some four-dimensional subspace into some seven-dimensional subspace? Set $(m, n, k, l, p)=(5,8,4,7,3)$ and compute

$$
\frac{l}{k}+\frac{m-k}{n-l}=\frac{7}{4}+\frac{1}{1}<3=p
$$


hence by the proposition the answer is no: there exist triples $\left(A_{1}, A_{2}, A_{3}\right)$ such that for all $U$ of dimension 4 we have $\sum A_{i} U=K^{8}$. This may not come as a surprise; however, it is not entirely obvious how to construct such a "generic" triple. For instance, we cannot choose them such that each $A_{i}$ is monomial in the sense that it maps every standard basis vector of $K^{5}$ to some multiple of a standard basis vector of $K^{8}$ : if this is the case, then the inequality $8 \cdot 2>5 \cdot 3$ implies that there is a basis vector $e_{i}$ of $K^{8}$ which is "hit only once" by some $A_{p}$ applied to some $e_{k}$. But then $U=\bigoplus_{l \neq k} K e_{l}$ is mapped into $\bigoplus_{j \neq i} K e_{j}$.

2. Do all triples of $5 \times 5$-matrices map some two-dimensional space into some threedimensional space? Set $(m, n, k, l, p)=(5,5,2,3,3)$ in the proposition. Now we find

$$
\frac{l}{k}+\frac{m-k}{n-l}=\frac{3}{2}+\frac{3}{2}=3=p,
$$

so we need a more detailed analysis. The cut-and-paste rank in the proposition is

$$
\mathrm{cp}^{(3)}(3,2,2,3) \text {, }
$$

which is $3 \cdot 2=6$ as one can cut a $3 \times 2$-matrix into $p=3$ rectangular pieces that can be put together without overlap to make up a $2 \times 3$-matrix. It follows that the dimension in the proposition is in fact pmn, i.e., that indeed, every triple of $5 \times 5$-matrices maps some two-dimensional space into some three-dimensional space. To prove this is a nice exercise for students in linear algebra. (It is also true in positive characteristic.)

To conclude the proof of Theorem 1 we need the following lemma.

Lemma 1 Let $V, W, m=\operatorname{dim} V, n=\operatorname{dim} W$, and the $C_{k}^{(p)}$ for $k=1, \ldots, m$ and $p \in \mathbb{N}$ be as in Theorem 1. Fix $k \in\{1, \ldots, m\}$ and let $l$ be the maximal integer with $l / k<n / m$. Then the following two statements are equivalent:

1. $C_{k}^{(p)}$ is not contained in $C_{k^{\prime}}^{(p)}$ for any $k^{\prime} \neq k$.

2. There exist a p-tuple $\left(A_{1}^{\prime}, \ldots, A_{p}^{\prime}\right) \in M_{l, k}^{p}$ such that

$$
\sum_{i} A_{i}^{\prime} K^{k}=K^{l}
$$

and

$$
\operatorname{dim}\left(\sum_{i} A_{i}^{\prime} U^{\prime}\right) \geq \frac{n}{m} \operatorname{dim} U^{\prime}
$$

for all proper subspaces $U^{\prime} \subsetneq K^{k}$; as well as a p-tuple $\left(A_{1}^{\prime \prime}, \ldots, A_{p}^{\prime \prime}\right) \in M_{n-l, m-k}$ such that

$$
l+\operatorname{dim}\left(\sum_{i} A_{i}^{\prime \prime} U^{\prime \prime}\right) \geq \frac{n}{m}\left(k+\operatorname{dim} U^{\prime \prime}\right)
$$

for all non-zero subspaces $0 \neq U^{\prime \prime} \subseteq K^{m-k}$.

Proof First suppose that the second condition is not satisfied, let $\left(A_{1}, \ldots, A_{p}\right)$ be in $C_{k}^{(p)}$, and let $V^{\prime}, W^{\prime}$ be subspaces of $V, W$ of dimensions $k, l$, respectively, such that $A_{i} V^{\prime} \subseteq W^{\prime}$ for all $i=1, \ldots, p$. 
Suppose that no $p$-tuple $\left(A_{i}^{\prime}\right)$ as above exists. Then for some $k^{\prime}<k$ the closed set consisting of all $\left(A_{i}^{\prime}\right) \in M_{l, k}^{p}$ for which there is a $k^{\prime}$-dimensional $U^{\prime}$ satisfying $\operatorname{dim}\left(\sum_{i} A_{i}^{\prime} U\right)<k^{\prime} n / m$ fills the entire space $M_{l, k}^{p}$. Taking for the $A_{i}^{\prime}$ the restrictions $\left.A_{i}\right|_{V^{\prime}}: V^{\prime} \rightarrow W^{\prime}$ we conclude that $C_{k}^{(p)} \subseteq C_{k^{\prime}}^{(p)}$.

Similarly, if no $p$-tuple $\left(A_{i}^{\prime \prime}\right)$ as above exists, then some $k^{\prime \prime} \in\{1, \ldots, m-k\}$ has the property that any $p$-tuple $\left(A_{i}^{\prime \prime}\right) \in M_{n-l, m-k}$ maps some $k^{\prime \prime}$-dimensional space into a space of dimension $<\left(k+k^{\prime \prime}\right) n / m-l$. In particular, for the $p$-tuple of induced linear maps $\overline{A_{i}}: V / V^{\prime} \rightarrow W / W^{\prime}$ there is a $k^{\prime \prime}$-dimensional space $U^{\prime \prime}$ for which $\operatorname{dim} \sum_{i} \overline{A_{i}} U^{\prime}<$ $\left(k+k^{\prime \prime}\right) n / m-l$. But then the preimage $U$ of $U^{\prime \prime}$ in $V$ is a space of dimension $k+k^{\prime \prime}$ that is mapped into a space of dimension $<\left(k+k^{\prime \prime}\right) n / m-l+l=\left(k+k^{\prime \prime}\right) n / m$, and we conclude that $C_{k}^{(p)} \subseteq C_{k+k^{\prime \prime}}^{(p)}$.

Conversely, suppose that $p$-tuples $\left(A_{i}^{\prime}\right)$ and $\left(A_{i}^{\prime \prime}\right)$ as above $d o$ exist. For $i=1, \ldots, p$ let $A_{i} \in M_{n, m}$ be the block matrix

$$
A_{i}=\left[\begin{array}{ll}
A_{i}^{\prime} & \\
& A_{i}^{\prime \prime}
\end{array}\right],
$$

and let $U$ be a subspace of $K^{m}$ unequal to $K^{k}$. Let $U^{\prime}$ be the intersection of $U$ with $K^{k}$ and let $U^{\prime \prime}$ be the projection of $U$ on $K^{m-k} \operatorname{along} K^{k}$. Then $\operatorname{dim} U=\operatorname{dim} U^{\prime}+\operatorname{dim} U^{\prime \prime}$ and one readily sees that

$$
\operatorname{dim}\left(\sum_{i} A_{i} U\right) \geq \operatorname{dim}\left(\sum_{i} A_{i}^{\prime} U^{\prime}\right)+\operatorname{dim}\left(\sum_{i} A_{i}^{\prime \prime} U^{\prime \prime}\right) .
$$

Now there are two possibilities: either $U^{\prime} \neq K^{k}$, or $U^{\prime}=K^{k}$ but $U^{\prime \prime} \neq 0$. In the first case one finds that the right-hand side is at least

$$
\frac{n}{m} \operatorname{dim} U^{\prime}+\frac{n}{m} \operatorname{dim} U^{\prime \prime}=\frac{n}{m} \operatorname{dim} U
$$

where we have used (6) for the first term, and (7) with $k$ and $l$ replaced by 0 for the second term - note that under this replacement (7) remains valid for $U^{\prime \prime} \neq 0$ by the choice of $l$, and becomes valid for $U^{\prime \prime}=0$, as well.

If, on the other hand, $U^{\prime}=K^{k}$ but $U^{\prime \prime} \neq 0$, then using (5) and (7) we find that the right-hand side in (8) is at least

$$
l+\operatorname{dim}\left(\sum_{i} A_{i}^{\prime \prime} U^{\prime \prime}\right) \geq \frac{n}{m}\left(k+\operatorname{dim} U^{\prime \prime}\right)=\frac{n}{m} \operatorname{dim} U .
$$

In other words, the pair $\left(K^{k}, K^{l}\right)$ is the only witness for the nilpotency of $\left(A_{1}, \ldots, A_{p}\right)$, and a fortiori this $p$-tuple lies in a unique $C_{k}^{(p)}$.

Proof (Proof of Theorem 1, part two). It is clear that if $p<q:=\left\lceil\frac{n}{m}\right\rceil$, then for any subspace $V^{\prime}$ of $V$ we have $\operatorname{dim}\left(\sum_{i=1}^{p} A_{i} V^{\prime}\right) \leq p \operatorname{dim} V^{\prime}<\frac{n}{m} \operatorname{dim} V^{\prime}$, so that all $C_{k}^{(p)}$ are equal to $M^{p}=\operatorname{Hom}(V, W)^{p}$. In other words: there are no invariants on $M^{p}$ for $p<q$.

Next suppose that $p \geq q+1$; then we have to show that there are no inclusions among the $C_{k}^{(p)}$. For every $k \in\{1, \ldots, m\}$ let $l_{k}:=\left\lceil k \frac{n}{m}\right\rceil-1$ denote the maximal $l \in\{0, \ldots, n-1\}$ with $\frac{l}{k}<\frac{n}{m}$. One readily verifies that

$$
1 \leq l_{k+1}-l_{k} \leq q \quad \text { for all } k \in\{1, \ldots, m-1\}
$$


(the first inequality follows from our standing assumption $n \geq m$ ). Fix $k \in\{1, \ldots, m\}$ and set $l:=l_{k}$, so that every $p$-tuple in $C_{k}^{(p)}$ maps some $k$-space into an $l$-space. We will prove the existence of $p$-tuples $\left(A_{i}^{\prime}\right) \in M_{l, k}^{p}$ and $\left(A_{i}^{\prime \prime}\right) \in M_{n-l, m-k}^{p}$ as in Lemma 1, so that $C_{k}^{(p)}$ is not contained in any $C_{k^{\prime}}^{(p)}$ with $k^{\prime} \neq k$.

To find the $A_{i}^{\prime}$ we show that for all $k^{\prime} \in\{1, \ldots, k-1\}$ and $l^{\prime} \in\{0, \ldots, l-1\}$ with $\frac{l^{\prime}}{k^{\prime}}<\frac{n}{m}$ the dimension of the set of $p$-tuples $\left(A_{1}^{\prime}, \ldots, A_{p}^{\prime}\right) \in M_{l, k}$ that map a $k^{\prime}$-space into an $l^{\prime}$-space is smaller than $p l k$. To this end we want to apply the sufficient condition of Proposition 2 with $m, n, k, l$ replaced by $k, l, k^{\prime}, l^{\prime}$, respectively. Compute therefore

$$
\frac{l^{\prime}}{k^{\prime}}+\frac{k-k^{\prime}}{l-l^{\prime}}<\frac{n}{m}+1 \leq q+1 \leq p
$$

where for the second term we used $l^{\prime} \leq l_{k^{\prime}}$ and the strict increasingness of the $l_{k}$. This shows the existence of $A_{1}^{\prime}, \ldots, A_{p}^{\prime}$ as required.

Similarly, to find the $A_{i}^{\prime \prime}$ we show that for all $k^{\prime} \in\{k+1, \ldots, m\}$ and $l^{\prime} \in\{l, \ldots, n-1\}$ with $\frac{l^{\prime}}{k^{\prime}}<\frac{n}{m}$ there exists a $p$-tuple $\left(A_{1}^{\prime \prime}, \ldots, A_{p}^{\prime \prime}\right) \in M_{m-k, n-l}$ that does not map any $\left(k^{\prime}-k\right)$-dimensional space into an $l^{\prime}-l$-dimensional space. Again, we apply the proposition, but now with $m, n, k, l$ replaced by $m-k, n-l, k^{\prime}-k, l^{\prime}-l$, respectively. Consider therefore the expression

$$
\frac{l^{\prime}-l}{k^{\prime}-k}+\frac{m-k^{\prime}}{n-l^{\prime}}
$$

As $l^{\prime} \leq l_{k^{\prime}}$ and $l=l_{k}$ the first term is at most $q$ by (9). On the other hand, as $l^{\prime}<\frac{n}{m} k^{\prime}$, the denominator of the second term satisfies

$$
n-l^{\prime}>n-\frac{n}{m} k^{\prime}=\frac{n}{m}\left(m-k^{\prime}\right) \geq m-k^{\prime},
$$

hence the second term is smaller than 1 . We conclude that

$$
p \geq q+1>\frac{l^{\prime}-l}{k^{\prime}-k}+\frac{m-k^{\prime}}{n-l^{\prime}},
$$

hence by Proposition 2 there exists a $p$-tuple $\left(A_{i}^{\prime \prime}\right)$ as required, and by Lemma 1 we conclude that $C_{k}^{(p)}$ is not contained in any $C_{k^{\prime}}^{(p)}$ with $k^{\prime} \neq k$. This concludes the case where $p>q$.

Finally, we assume that $p=q$. First suppose that there exists a $k \in\{1, \ldots, m-1\}$ with $l_{k+1}-l_{k}=q$. Then any $q$-tuple $\left(A_{1}, \ldots, A_{q}\right) \in C_{k}^{(q)}$ maps a $k$-space into an $l_{k}$-space, and adding one arbitrary dimension to that $k$-space yields a $(k+1)$-space mapped by all $A_{i}$ into a space of dimension $l_{k}+q=l_{k+1}$. In other words, we have $C_{k}^{(q)} \subseteq C_{k+1}^{(q)}$, so that there are indeed inclusions among the $C_{k}^{(q)}$. Next suppose that no such $k$ exists. Then we have

$$
n-1=l_{m} \leq l_{1}+(m-1)(q-1)=m(q-1)<m \frac{n}{m}=n,
$$

so that $n=m(q-1)+1$, where $q \geq 2$. In this case $l_{k}=(q-1) k$ for all $k$, and for $q>2$ the inequalities

$$
\frac{l_{k^{\prime}}}{k^{\prime}}+\frac{k-k^{\prime}}{l_{k}-l_{k^{\prime}}}=(q-1)+\frac{1}{q-1}<q \quad \text { for } k^{\prime}<k
$$


and

$$
\frac{l_{k^{\prime}}-l_{k}}{k^{\prime}-k}+\frac{m-k^{\prime}}{n-l_{k^{\prime}}}=(q-1)+\frac{m-k^{\prime}}{(q-1)\left(m-k^{\prime}\right)+1}<q \quad \text { for } k^{\prime}>k
$$

readily imply that the construction of the $A_{i}$ above still works to show that $C_{k}^{(q)}$ is not contained in any other $C_{k^{\prime}}^{(q)}$. The last case to be considered is $q=2$ and $n=m+1$. Then $l_{k}=k$ for all $k$, and any pair of matrices mapping a $k$-space into a $k$-space also maps a $(k-1)$-space into a $(k-1)$-space, so that the null-cone on $q=2$ copies is irreducible.

We should point out that, although Theorem 1 does settle the question of when all irreducible components of the null-cone in $\operatorname{Hom}(V, W)^{p}$ become visible, it does not conclusively describe the irreducible components in the case where $p=q:=\lceil n / m\rceil$. Frankly, we do not fully understand the null-cone in this representation: although an easy dimension count shows that $\mathrm{SL}(V) \times \mathrm{SL}(W)$ cannot have a dense orbit on $\operatorname{Hom}(V, W)^{q}$, so that the null-cone does not fill up the entire space, it seems hard to predict which inclusions there exist among the $C_{k}^{(q)}$. The only thing that we venture to say in general is that there seem to be many inclusions when $n$ is close or equal to $q m$ and few inclusions when $q \geq 3$ and $n$ is close to $(q-1) m$. In concrete cases, however, Lemma 1 and Proposition 2 allow one to determine explicitly which of the $C_{k}^{(q)}$ are maximal. We have thus reduced the problem of determining the irreducible components of the null-cone on $q$ copies to the computation of cut-and-paste ranks - as this is the only non-trivial thing one has to do to apply Lemma 1 and Proposition 2. We conclude the discussion of the null-cone on $\operatorname{Hom}(V, W)^{q}$ with a few examples.

\section{Example 2.}

1. If $n=q m$, then $C_{k}^{(q)}$ is the set of $q$-tuples mapping some $k$ dimensional space into a $(k q-1)$-dimensional space. Clearly, they form a chain $C_{1}^{(q)} \subseteq C_{2}^{(q)} \subseteq \cdots \subseteq C_{m}^{(q)}$, so that the null-cone is equal to the last term and irreducible.

2. Let $m=4, n=6, p=q=2$. Then $C_{k}:=C_{k}^{(2)}$ is the set of pairs of linear maps $K^{4} \rightarrow K^{6}$ mapping some $k$-dimensional space into an $l_{k}$-dimensional space, where $l_{k}=1,2,4,5$ for $k=1,2,3,4$, respectively. One has the inclusions $C_{1}, C_{2}, C_{4} \subseteq C_{3}$, so that the null-cone is equal to $C_{3}$ and irreducible (we do not claim that these are all inclusions among the $C_{i}$ ). Indeed, the inclusion $C_{2} \subseteq C_{3}$ is easy. To see that $C_{4} \subseteq C_{3}$ we apply Proposition 2 with $(m, n, k, l, p)$ equal to $(4,5,3,4,2)$ : the dimension of the variety $Q$ there equals

$$
40-2 \cdot 1+\mathrm{cp}^{(2)}(1,3,1,2)=40,
$$

so that every pair of $5 \times 4$-matrices maps some three-dimensional space into a four-dimensional space (this can, of course, also be seen directly).

Similarly, to see that $C_{1} \subseteq C_{3}$ we apply Proposition 2 with $(m, n, k, l, p)$ equal to $(3,5,2,3,2)$. The dimension of $Q$ is now

$$
30-1 \cdot 2+\mathrm{cp}^{(2)}(1,2,2,1)=30,
$$

so that every pair of $5 \times 3$-matrices maps some two-dimensional space into a three-dimensional space. Applying, as in Lemma 1, this fact to the linear maps induced by a pair $\left(A_{1}, A_{2}\right) \in C_{1}$, which go from a three-dimensional quotient space to a five-dimensional quotient space, we find that $C_{1} \subseteq C_{3}$. 
3. Let $m=5, n=12, p=3$. Then $l_{k}=2,4,7,9,11$ for $k=1,2,3,4,5$, respectively; write $C_{k}:=C_{k}^{(3)}$. We readily find $C_{2} \subseteq C_{3}$. We claim that no $C_{k}$ with $k \neq 2$ is contained in any $C_{k^{\prime}}$ with $k^{\prime} \neq k$. Again, one can prove this using Lemma 1 and Proposition 2. Indeed, it turns out that for $k=1,3,4,5$ the sufficient criterion

$$
3=p>l^{\prime} / k^{\prime}+\left(m^{\prime}-k^{\prime}\right) /\left(n^{\prime}-l^{\prime}\right)
$$

of Proposition 2 is verified for all values $m^{\prime}:=k, n^{\prime}:=l_{k}, k^{\prime}<k, l^{\prime}:=l_{k^{\prime}}$ as well as for all values $m^{\prime}:=m-k, n^{\prime}:=n-l_{k}, 1<k^{\prime} \leq m-k, l^{\prime}:=l_{k+k^{\prime}}-l_{k}$. Using Lemma 1 , this proves that the null-cone has four irreducible components, namely $C_{1}, C_{3}, C_{4}, C_{5}$.

As promised in the Introduction, we now investigate when the polarisations of invariants on one copy of $\operatorname{Hom}(V, W)$ define the null-cone on $p$ copies. This question is interesting only in the case where there are non-trivial invariants on one copy-hence if $\operatorname{dim} V=\operatorname{dim} W$, in which case we may as well assume $V=W$. The invariant ring of $\operatorname{SL}(V) \times \operatorname{SL}(V)$ on $\operatorname{End}(V)$ is generated by the determinant; this readily follows from the fact that every invertible matrix $A$ has the matrix $\operatorname{diag}(\operatorname{det} A, 1, \ldots, 1)$ in its orbit. Note that by Theorem 1 the $p$-tuples in the null-cone on $\operatorname{End}(V)^{p}$ are precisely those whose span in $\operatorname{End}(V)$ is a "compression space" in the sense that it maps some subspace of $V$ into a strictly smaller subspace; see [7] for this terminology. On the other hand, the $p$-tuples on which all polarisations of det vanish are those that span a "singular space", i.e., a vector space in which every linear map is singular. Hence, the polarisations of det define the null-cone on $\operatorname{End}(V)^{p}$ if and only if every singular space in $\operatorname{End}(V)$ spanned by $p$ matrices is is a compression space. See [5] for interesting small examples of singular non-compression spaces.

Theorem 2. The null-cone in $\operatorname{End}(V)^{p}$ is defined by the polarisations of det if and only if $\operatorname{dim} V \leq 2$ or $p \leq 2$.

Proof (Proof of Theorem 2). The result for $p=2$ follows from the KroneckerWeierstrass theory of matrix pencils, see [8]; for completeness we include a short proof in our terminology. By Theorem 1 we have to show that if $A, B \in \operatorname{End}(V)$ satisfy $\operatorname{det}(s A+t B)=0$ for all $s, t \in K$, then there exists a witness $V^{\prime}, W^{\prime} \subseteq V$ for the nilpotency of $(A, B)$. Indeed, regarding $s, t$ as variables, $s A+t B$ has a non-zero vector $u(s, t)$ in $K[s, t] \otimes_{K} V$ in its kernel. But then any non-zero homogeneous component of $u(s, t)$, say of degree $d$, is also annihilated by $s A+t B$; hence we find $u_{0}, \ldots, u_{d} \in V$ such that $(s A+t B)\left(s^{d} u_{0}+s^{d-1} t u_{1}+\cdots+t^{d} u_{d}\right)=0$, where we may assume that $u_{0} \neq 0$. Taking the of coefficients of $s^{d+1}, s^{d} t, \ldots, t^{d+1}$, we find

$$
A u_{0}=0, A u_{1}=-B u_{0}, \ldots, A u_{d}=-B u_{d-1}, \quad \text { and } B u_{d}=0 .
$$

But then every element of $K A+K B$ maps the space $V^{\prime}:=\sum_{i} K u_{i}$ into the space $U^{\prime}:=\sum_{i} K A u_{i}$, which is strictly smaller because $A u_{0}=0$ while $u_{0} \neq 0$.

The statement for $\operatorname{dim} V=2$ is easy: in a linear space of matrices of rank $\leq 1$ either all matrices have the same image, or all matrices have the same kernel (otherwise the space contains an $A=\lambda \otimes u$ and a $B=\mu \otimes v$ such that both $\lambda, \mu \in V^{*}$ and $u, v \in V$ are linearly independent - but then $A+B$ has rank 2). Now suppose that $m, n \geq 3$. To show that the null-cone in $\operatorname{End}(V)^{3}$ is then not defined by the polarisations of det, it suffices to construct a three-dimensional singular subspace of $\operatorname{End}(V)$ for which there do not exist $V^{\prime}, W^{\prime}$ as above. The space 


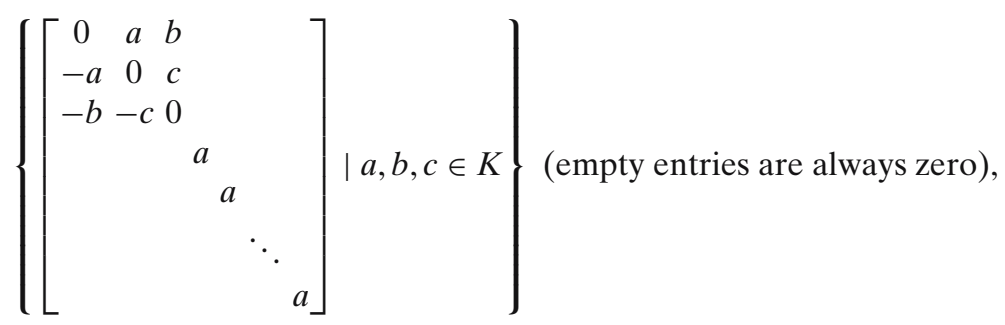

is such a space, as one easily verifies.

\section{$3 \mathrm{SL}(V)$ on symmetric bilinear forms}

The group $\operatorname{SL}(V)$ acts on bilinear forms as follows: if $\alpha$ is a bilinear form and $g \in \operatorname{SL}(V)$, then $(g \alpha)(v, w)=\alpha\left(g^{-1} v, g^{-1} w\right)$. It will be convenient to associate to every bilinear a linear map as follows: we fix, once and for all, a non-degenerate, symmetric bilinear form (.,.) on $V$, and denote the transpose of $A \in \operatorname{End}(V)$ relative to this form by $A^{t}$. If $\alpha$ is a bilinear form on $V$, then we associate to $\alpha$ a linear map $A$ by the requirement that $\alpha(x, y)=(x, A y)$ for all $x, y \in V$. Then $g$ acts on $A$ by $g \cdot A:=\left(g^{-1}\right)^{t} A g^{-1}$. Note that the image of $\operatorname{SL}(V)$ in $\operatorname{GL}(\operatorname{End}(V))$ under this representation is contained in the image of $\operatorname{SL}(V) \times \operatorname{SL}(V)$ under the representation of Sect. 2 .

If $\alpha$ is a symmetric or skew symmetric bilinear form on $V$, and if $U$ is a subspace of $V$, then we will call the space $\{v \in V \mid \alpha(v, U)=0\}$ the $\alpha$-perp of $U$. If $A$ is the linear map associated to $\alpha$, then this also the (., .)-perp of $A U$.

As in Sect. 2 the invariants of $\operatorname{SL}(V)$ on $S^{2}\left(V^{*}\right)$ are generated by the determinant of (the linear map associated to) the form, and the null-cone on one copy is therefore the irreducible variety of singular forms.

Theorem 3. For $p \geq 2$ and $n:=\operatorname{dim} V$, the null-cone of $\operatorname{SL}(V)$ on $S^{2}\left(V^{*}\right)^{p}$ has $\left\lfloor\frac{n+1}{2}\right\rfloor$ irreducible components given by

$$
\begin{aligned}
C_{k}^{(p)}:= & \left\{\left(\alpha_{1}, \ldots, \alpha_{p}\right) \mid \exists U \subseteq W \subseteq V: \operatorname{dim} U=k, \operatorname{dim} W=n-k+1,\right. \text { and } \\
& \left.\alpha_{i}(U, W)=0 \quad \text { foralli }=1, \ldots, p\right\}, \quad k=1, \ldots,\left\lfloor\frac{n+1}{2}\right\rfloor .
\end{aligned}
$$

In contrast to our proof for tuples of matrices, we will give explicit pairs of symmetric forms representing the various components of the null-cone; for this the following lemma is useful.

Lemma 2. Let $m, n, k$ be non-negative integers and let $\pi_{1}, \ldots, \pi_{p}$ be partially defined strictly increasing functions $\{1, \ldots, m\} \rightarrow\{1, \ldots, n\}$, that is, every $\pi_{l}$ is defined on a subset $\operatorname{dom}\left(\pi_{l}\right)$ of $\{1, \ldots, m\}$ and satisfies

$$
i<j \Rightarrow \pi_{l}(i)<\pi_{l}(j) \text { whenever the right-hand side is defined. }
$$

For $l=1, \ldots, p$ let $A_{l}: K^{m} \rightarrow K^{n}$ be a linear map mapping $e_{i}$ to a non-zero multiple of $e_{\pi_{l}(i)}$ if $\pi_{l}$ is defined at $i$, and to zero otherwise. Let $U$ be a subspace of $K^{m}$ and set

$$
\operatorname{gr} U:=\left\{i \in\{1, \ldots, m\} \mid U \cap\left(e_{i}+\left\langle e_{1}, \ldots, e_{i-1}\right\rangle_{K}\right) \neq \emptyset\right\} .
$$


Then

$$
\operatorname{dim} \sum_{l} A_{l} U \geq\left|\bigcup_{l} \pi_{l}\left(\operatorname{gr} U \cap \operatorname{dom} \pi_{l}\right)\right|
$$

We will call a $p$-tuple $\left(A_{1}, \ldots, A_{p}\right)$ of linear maps as in this lemma standard.

Proof. We have $|\operatorname{gr}(U)|=\operatorname{dim} U$, and defining gr $W$ for subspaces $W$ of $K^{n}$ in a similar way the conditions on the $A_{i}$ guarantee that

$$
\operatorname{gr}\left(\sum_{l} A_{l} U\right) \supseteq \bigcup_{l} \pi_{l}\left(\operatorname{gr} U \cap \operatorname{dom} \pi_{l}\right),
$$

whence the lemma follows immediately.

Proof (Proof of Theorem 3). Suppose that $\left(\alpha_{1}, \ldots, \alpha_{p}\right)$ lies in the null-cone, and let $A_{i}$ be the linear map associated to $\alpha_{i}$. Then $\left(A_{1}, \ldots, A_{p}\right)$ lies in the null-cone of SL $(V)$ acting on $\operatorname{End}(V)$ as indicated above and, a fortiori, in the null-cone of $\operatorname{SL}(V) \times \operatorname{SL}(V)$ on $\operatorname{End}(V)$ discussed in Section 2. Hence by Theorem 1 there exist subspaces $U^{\prime}$ and $W^{\prime}$ of $V$ with $\operatorname{dim} W^{\prime}=n-\operatorname{dim} U^{\prime}+1$ and such that every $A_{i}$ maps $U^{\prime}$ into the (., .)-perp of $W^{\prime}$ relative to (.,.) (So $W^{\prime}$ here is the (.,.)-perp of the space $W^{\prime}$ in Theorem 1.) But then $\alpha_{i}(w, u)=\left(w, A_{i} u\right)=0$ for all $u \in U^{\prime}$ and $w \in W^{\prime}$. Now set $U:=U^{\prime} \cap W^{\prime}$ and $W:=U^{\prime}+W^{\prime}$. Then clearly $U \subseteq W, \operatorname{dim} U+\operatorname{dim} W=\operatorname{dim} U^{\prime}+\operatorname{dim} W^{\prime}=n+1$, and $\alpha_{i}(U, W)=0$ for all $i$.

The $C_{k}^{(p)}$ are closed and irreducible as usual (see Sect. 1), and so it only remains to check that there are no inclusions among them for $p \geq 2$. To this end, let $k \in$ $\left\{1, \ldots,\left\lfloor\frac{n+1}{2}\right\rfloor\right\}$; we will construct a pair $(\alpha, \beta) \in C_{k}^{(2)}$ that does not lie in any $C_{k^{\prime}}^{(2)}$ with $k \neq k^{\prime}$. Take $V=K^{n}$ and $(x, y):=\sum_{i=1}^{n} x_{i} y_{n+1-i}$, so that transposition relative to this form corresponds to reflection of the matrix in the "skew diagonal"; we will refer to this symmetric form as the skew diagonal symmetric form. Now take the standard pair $(A, B)$ for which

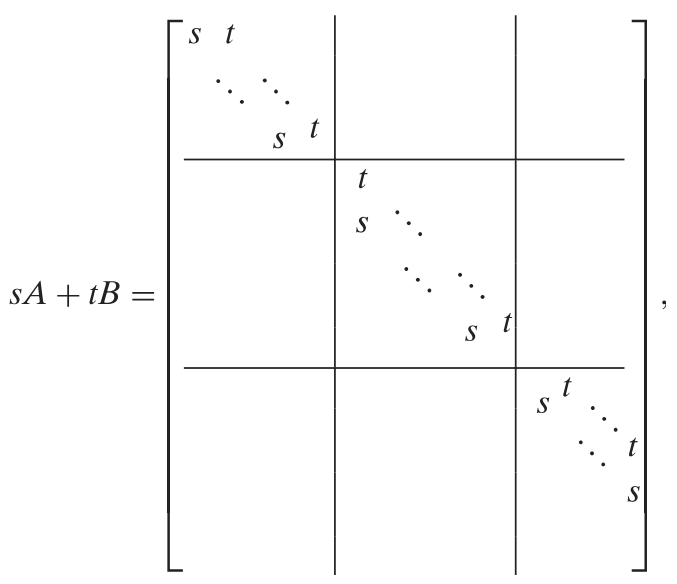

where the diagonal block sizes are, from top left to bottom right, $(k-1) \times k,(n-$ $2 k+1) \times(n-2 k+1)$, and $k \times(k-1)$. Let $\alpha$ and $\beta$ be the forms defined by $A$ and $B$, 
respectively. Now if $U$ and $W$ are subspaces of $K^{n}$ with $\operatorname{dim} U+\operatorname{dim} W=n+1$ and $\alpha(U, W)=\beta(U, W)=0$, then one finds $\operatorname{dim}(A U+B U)<\operatorname{dim} U$. But by Lemma 2 the only pair of subspaces of $K^{n}$ having this property are $U=\left\langle e_{1}, \ldots, e_{k}\right\rangle_{K}$ and $W=\left\langle e_{1}, \ldots, e_{k}, \ldots, e_{n-k+1}\right\rangle_{K}$. This shows that $(U, W)$ is the unique witness for the nilpotency of $(\alpha, \beta)$, and hence $(\alpha, \beta)$ does not lie in any other component $C_{k^{\prime}}^{(2)}$.

We now proceed with our second fundamental problem: for which $p, n$ is the nullcone on $p$-tuples of symmetric bilinear forms on $V$ defined by the polarisations of det? Suppose that $\left(\alpha_{1}, \ldots, \alpha_{p}\right)$ lies in $C_{k}^{(p)}$, and that $U$ and $W$ are a witness of its nilpotency as in Theorem 3. A dimension argument shows that $U$ must intersect the radical of each $\alpha_{i}$ non-trivially; in particular, if $\alpha_{i}$ has rank $n-1$, then its radical is contained in $U$, and $W$ is precisely the $\alpha_{i}$-perp of $U$.

Suppose now that all $\alpha_{i}$ have rank $n-1$. Then a geometric interpretation of $U, W$ as in the theorem is the following: $\mathbb{P} U$ is a linear subspace of $\mathbb{P} V$ common to all quadrics $Q_{i}=\left\{x \in \mathbb{P} V \mid \alpha_{i}(v, v)=0\right\}$ and containing their radicals, and for each $i, \mathbb{P} W$ is the space tangent to $Q_{i}$ at all of $\mathbb{P} U$. For example, if $n=4$ and $p=2$, then a pair $\left(\alpha_{1}, \alpha_{2}\right)$ of rank 3 forms lies in $C_{1}^{(2)}$ if and only if $\alpha_{1}$ and $\alpha_{2}$ have the same radical (a projective point); if $\left(\alpha_{1}, \alpha_{2}\right) \notin C_{1}^{(2)}$, then the pair lies in $C_{2}^{(2)}$ if and only if the quadrics $Q_{1}, Q_{2}$ are tangent along the (projective) line through their radicals. This interpretation yields a nice proof of the following theorem.

Theorem 4. The null-cone on $S^{2}\left(V^{*}\right)^{p}$ is defined by the polarisations of det if and only if $\operatorname{dim}(V) \leq 4$ or $p \leq 2$.

Proof (Proof of Theorem 4). On $p=2$ copies the null-cone is defined by the polarisations of the determinant. This follows either from the Kronecker-Weierstrass theory of pencils of forms [8] or from a direct construction of $U$ and $W$ as in Theorem 3 for any two-dimensional space of singular forms.

Next we prove that for $n \leq 4$ the null-cone on any number $p$ of copies is defined by the polarisations of det, or, in other words, that any space $\mathcal{A}$ of singular symmetric bilinear forms is spanned by a tuple $\left(\alpha_{1}, \ldots, \alpha_{p}\right)$ lying in some $C_{k}^{(p)}$; slightly inaccurately, we will then say that $\mathcal{A}$ lies in $C_{k}$. Note that we need only prove this for maximal spaces of singular forms; in particular, we may assume that $\mathcal{A}$ contains forms of rank $n-1$, because if it does not, we may add any rank 1 form to $\mathcal{A}$ without creating nondegenerate forms. In what follows we heavily use the fact that any two-dimensional space of singular forms does already lie in some $C_{k}$.

For $n=2$, the quadric of a rank 1 form is a point on the projective line $\mathbb{P} V$. As for any two non-zero forms in $\mathcal{A}$ this point coincides, it is the same for all forms in $\mathcal{A}$. Hence $\mathcal{A}$ lies in $C_{1}$.

For $n=3$, the quadric of a rank 2 form $\alpha$ is the union of two lines in the projective plane $\mathbb{P} V$, whose intersection is the radical of $\alpha$. If the radicals of any two forms in $\mathcal{A}$ of rank 2 coincide, then $\mathcal{A}$ lies in $C_{1}$; suppose, therefore, that there exist forms $\alpha_{0}, \alpha_{1}$ in $\mathcal{A}$ of rank 2 whose radicals are distinct. We have $\left(\alpha_{0}, \alpha_{1}\right) \in C_{2}$, so that their quadrics $Q_{0}$ and $Q_{1}$ have a line $L$ in common (see Fig. 2). Now a generic element $\beta \in \mathcal{A}$ has rank 2, does not have the same radical as $\alpha_{0}$ or $\alpha_{1}$, and its quadric $Q_{\beta}$ is not the union of the non-common lines of $Q_{0}$ and $Q_{1}$. But $Q_{\beta}$ must have lines in common with both $Q_{0}$ and $Q_{1}$, and therefore it contains $L$. But then $L$ is isotropic relative to all forms in $\mathcal{A}$, and $\mathcal{A}$ lies in $C_{2}$.

For $n=4$, suppose that there exist forms $\alpha_{0}, \alpha_{1} \in \mathcal{A}$ of rank 3 whose radicals do not coincide (otherwise $\mathcal{A}$ lies in $C_{1}$ ). The corresponding quadrics $Q_{0}, Q_{1} \subseteq \mathbb{P} V$ 
Fig. 2 Proof of Theorem 4 for $n=3$
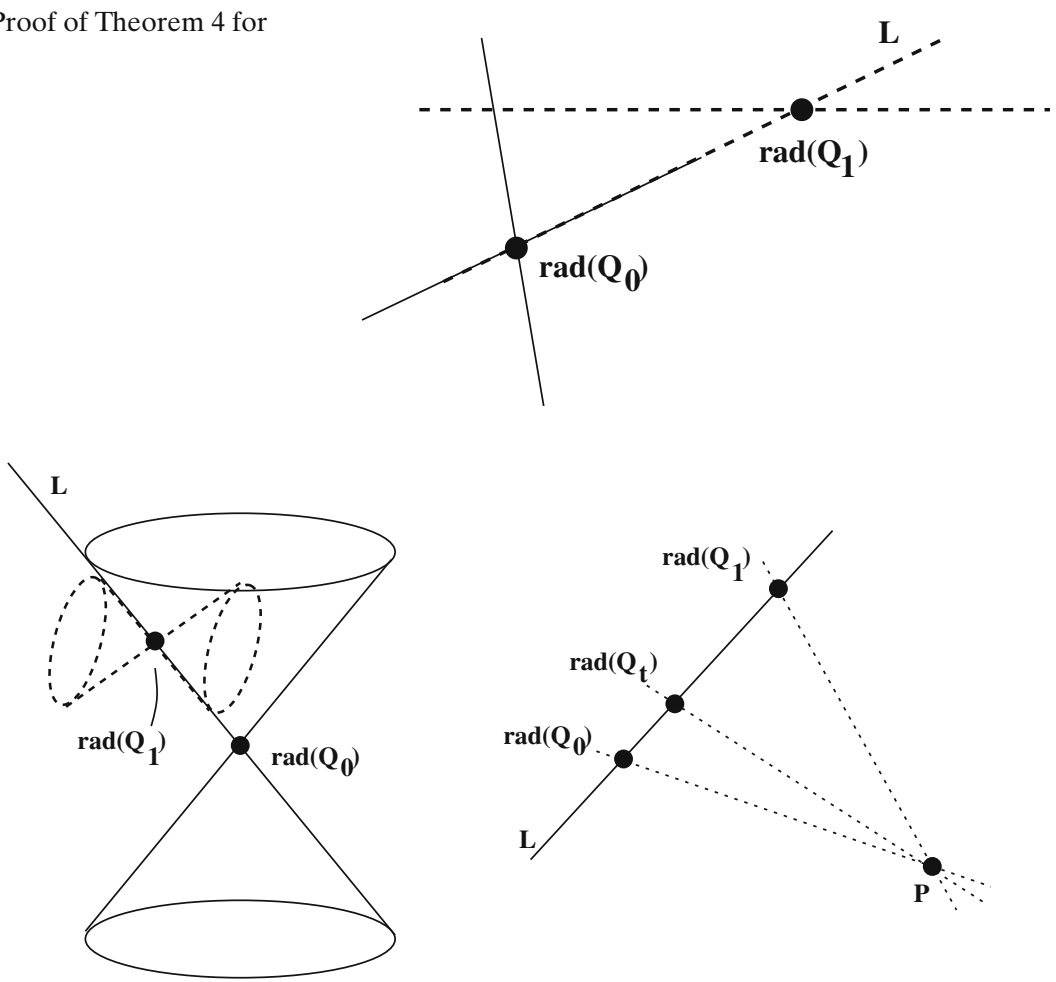

Fig. 3 Proof of Theorem 4 for $n=4$

are tangent along the line $L$ connecting their radicals (see Fig. 3, left). For $t \in K$ set $\alpha_{t}:=(1-t) \alpha_{0}+t \alpha_{1}$ and

$$
T:=\left\{t \in K \mid \operatorname{rk}\left(\alpha_{t}\right)=3\right\}
$$

For each $t \in T$, the quadric $Q_{t}$ of $\alpha_{t}$ is tangent to $Q_{0}$ along $L$, and its radical lies on $L$; the set of all radicals thus obtained forms a dense set of $L$.

If all rank 3 forms in $\mathcal{A}$ have their radicals on $L$, then their quadrics are all tangent to $Q_{0}$ along $L$ and $\mathcal{A}$ lies in $C_{2}$. Suppose, on the other hand, that there exists a rank 3 form $\beta \in \mathcal{A}$ whose radical does not lie on $L$. Then its quadric $Q_{\beta}$ is tangent to each $Q_{t}$ with $t \in T$ along the line connecting $P:=\mathbb{P} \operatorname{rad}(\beta)$ and $\mathbb{P} \operatorname{rad}\left(\alpha_{t}\right)$; in particular, $Q_{\beta}$ contains all lines connecting $P$ with a dense subset of $L$ (see Fig. 3, right). The closure of the union of these lines - the projective plane spanned by $L$ and $P$-is therefore contained in $Q_{\beta}$. Hence, the pre-image in $V$ of this plane is a three-dimensional $\beta$-isotropic space-but this contradicts the assumption that $\operatorname{rk}(\beta)=3$.

Finally, we need to show that if $n \geq 5$ and $p \geq 3$, then the null-cone is not defined by the polarisations of det. To this end, take for (.,.) on $V=K^{n}$ the orthogonal sum of the skew diagonal symmetric form on $K^{5}$ and the skew diagonal symmetric form on $K^{n-5}$. Consider the triple $\left(\alpha_{1}, \alpha_{2}, \alpha_{3}\right)$ of bilinear forms on $K^{n}$ for which the linear map associated to $s \alpha+t \beta+u \gamma$ relative to (.,.) equals 


$$
s A_{1}+t A_{2}+u A_{3}=\left[\begin{array}{ccc|cc|c}
s & t & 0 & 0 & 0 & \\
0 & s & t & 0 & 0 & \\
\hline-u & 0 & 0 & t & 0 & \\
0 & 2 u & 0 & s & t & \\
0 & 0 & -u & 0 & s & \\
\hline & & & & s I_{n-5}
\end{array}\right]
$$

A direct computation shows that $\operatorname{det}\left(s A_{1}+t A_{2}+u A_{3}\right)=0$. On the other hand, by Lemma 2 there exists no subspace $U$ of $K^{n}$ with $\operatorname{dim}\left(\sum_{i} A_{i} U\right)<\operatorname{dim} U$. We conclude that $\left(\alpha_{1}, \alpha_{2}, \alpha_{3}\right)$ is not nilpotent, and this concludes the proof of Theorem 4.

Remark 4. The description of the null-cone in Theorem 3 already appears in [17, Theorem 0.1(ii)]. However, Wall claims in Corollary 1 of loc. cit. that the null-cone on any number of copies is defined by the polarisations of det-which, as we have just seen, is only the case for $n<5$.

\section{$4 \mathrm{SL}(V)$ on skew-symmetric forms}

Our results for skew-symmetric forms are similar to those for symmetric forms, except that the irreducible components of the null-cone become visible only from 3 or 4 copies onwards. Recall that if $n:=\operatorname{dim}(V)$ is odd, then all skew bilinear forms are singular and there are no invariants on one copy of $\bigwedge^{2}\left(V^{*}\right)$, so that the null-cone is the whole space. If $n$ is even, then the invariant ring is generated by the Pfaffian and the null-cone is irreducible.

Theorem 5. The null-cone $\operatorname{SL}(V)$ on $\bigwedge^{2}\left(V^{*}\right)^{p}$ is equal to

$$
\begin{aligned}
& \left\{\left(\alpha_{1}, \ldots, \alpha_{p}\right) \mid \exists U \subseteq W \subseteq V \text { with } \operatorname{dim} U+\operatorname{dim} W=n+1\right. \\
& \text { and } \left.\alpha_{i}(U, W)=0 \text { for all } i=1, \ldots, p\right\} .
\end{aligned}
$$

Let $C_{k}^{(p)}$ denote the subset of the null-cone where $U$ can be chosen of dimension $k\left(=1, \ldots,\left\lceil\frac{n}{2}\right\rceil=: q\right)$. Then the irreducible components of the null-cone are as follows.

1. If $n=2 q \geq 2$ is even, then the null-cone on $p=2$ copies is $C_{q}^{(2)}$ (hence irreducible), while the null-cone on $p \geq 3$ copies has precisely $q$ components, namely $C_{k}^{(p)}$ for $k=1, \ldots, q$.

2. If $n=2 q-1 \geq 3$ is odd, then the null-cone on $p=2$ copies is all of $\wedge^{2}\left(V^{*}\right)^{p}$; on $p=3$ copies there are non-trivial invariants, and the components of the null-cone are precisely the $C_{k}^{(3)}$ with $k \in\{1,2, \ldots, q-4, q\}$ (in particular, for $n \leq 7$ the nullcone is irreducible); on $p=4$ copies the components of the null-cone are precisely the $C_{k}^{(4)}$ with $k \in\{1,2, \ldots, q-3, q\}$ (in particular, for $n \leq 5$ the null-cone is irreducible); and on $p \geq 5$ copies the components of the null-cone are precisely the $C_{k}^{(p)}$ with $k \in\{1,2, \ldots, q-2, q\}$ (in particular, for $n \leq 3$ the null-cone is irreducible).

For the proof of this theorem we need a result from [3], which uses the following notation: $d(n, p)$ is the minimum, taken over all $p$-tuples $\left(\alpha_{1}, \ldots, \alpha_{p}\right)$ of skew bilinear forms on $K^{n}$, of the maximal dimension of a subspace that is isotropic with respect to all $\alpha_{i}$. In other words, $d(n, p)$ is the maximal dimension of a common isotropic subspace of a generic $p$-tuple of skew bilinear forms on $K^{n}$. 
Theorem 6 ([3, Main Theorem $]) \cdot d(n, p)=\left\lfloor\frac{2 n+p}{p+2}\right\rfloor$.

Corollary 1. For $n=0,2,4,6$ any triple of skew bilinear forms on $K^{n}$ has a common isotropic subspace of dimension $n / 2$. On the other hand, for all odd $n \geq 3$ and for all even $n \geq 8$ there exist triples $\left(\alpha_{1}, \alpha_{2}, \alpha_{3}\right)$ of skew bilinear forms on $K^{n}$ for which there are no subspaces $0 \subsetneq U \subseteq W$ of $K^{n}$ with $\operatorname{dim} U+\operatorname{dim} W=n$ and $\alpha_{i}(U, W)=0$ for all $i$.

Proof. The first statement is immediate from Theorem 6. Now let $n=2 q \geq 8$ be even, fix $k \in\{1, \ldots, q\}$, and suppose that for any triple $\alpha_{1}, \alpha_{2}, \alpha_{3}$ of skew bilinear forms on $K^{n}$ there exist subspaces $0 \neq U \subseteq W$ of $K^{n}$ with $\operatorname{dim} U=k=n-\operatorname{dim} W$ and $\alpha_{i}(U, W)=0$ for all $i=1,2,3$. The induced forms $\bar{\alpha}_{i}, i=1,2,3$, on the space $W / U$ of dimension $2(q-k)$ have a common isotropic subspace $U^{\prime} \subseteq W / U$ of dimension $d(2(q-k), 3)$, by definition of the latter quantity. The pre-image of $U^{\prime}$ in $W$ is then isotropic relative to all $\alpha_{i}$ and has dimension $d(2(q-k), 3)+k$. We thus find the inequality $d(2 q, 3) \geq d(2(q-k), 3)+k$, which by Theorem 6 reads

$$
\left\lfloor\frac{4 q+3}{5}\right\rfloor \geq\left\lfloor\frac{4(q-k)+3}{5}\right\rfloor+k=\left\lfloor\frac{4 q+3}{5}+\frac{k}{5}\right\rfloor .
$$

For $n=2 q=8$, however, this inequality does not hold for any $k \in\{1,2,3,4\}$. For $n=2 q=10$ the only $k \in\{1, \ldots, 5\}$ for which it holds is $k=1$, but it is easy to construct a triple of bilinear forms on $K^{10}$ for which there are no $U, W$ as above of dimensions 1,9-indeed, one can use for this the construction that follows.

Suppose that $n=2 q \geq 12$, and note that inequality (10) can only hold for $k \leq 5$. On the other hand, let $\alpha_{1}, \alpha_{2}, \alpha_{3}$ be the skew bilinear forms on $K^{n}$ corresponding to the triple $\left(A_{1}, A_{2}, A_{3}\right)$ of matrices, standard in the sense of Lemma 2, satisfying

$$
t_{1} A_{1}+t_{2} A_{2}+t_{3} A_{3}=\left[\begin{array}{cccccccc}
t_{2} & t_{3} & & & & & \\
t_{1} & t_{2} & \ddots & & & & \\
& \ddots & \ddots & t_{3} & & & \\
& & t_{1} & t_{2} & & & \\
& & & -t_{2} & -t_{3} & \\
& & & -t_{1} & \ddots & \ddots \\
& & & & \ddots & -t_{2}-t_{3} \\
& & & & & -t_{1}-t_{2}
\end{array}\right] .
$$

Using Lemma 2 one verifies that any subspace $U$ of $K^{n}$ satisfying $\operatorname{dim}\left(A_{1} U+A_{2} U+\right.$ $\left.A_{3} U\right) \leq \operatorname{dim} U$ has dimension $0, n / 2$, or $n$. In particular, we should have $k \in\{0, q, n\}-$ but we saw above that $1 \leq k \leq 5$, a contradiction.

We conclude that for $n=2 q \geq 8$ and fixed $k \in\{1, \ldots, q\}$ there exist triples $\left(\alpha_{1}, \alpha_{2}, \alpha_{3}\right)$ of skew bilinear forms on $K^{n}$ for which there are no subspaces $U \subseteq W$ of $K^{n}$ with $\operatorname{dim} U=k=n-\operatorname{dim} W$ and $\alpha_{i}(U, W)=0$ for all $i$. As the non-existence of such a pair $U, W$ with $\operatorname{dim} U=k$ is an open condition on the triple $\left(\alpha_{1}, \alpha_{2}, \alpha_{3}\right)$, there also exist triples for which there is no pair $(U, W)$ with $U$ of any dimension. This proves the corollary for even $n$.

For $n=2 q-1 \geq 3$ odd we can construct $\alpha_{1}, \alpha_{2}, \alpha_{3}$ explicitly by a construction similar to that above: choose them corresponding to a standard triple $\left(A_{1}, A_{2}, A_{3}\right)$ of matrices satisfying 


$$
t_{1} A_{1}+t_{2} A_{2}+t_{3} A_{3}=\left[\begin{array}{ccccccc}
t_{2} & t_{3} & & & & & \\
t_{1} & \ddots & \ddots & & & & \\
& \ddots & t_{2} & t_{3} & & & \\
& t_{1} & 0 & -t_{3} & & \\
& & -t_{1} & -t_{2} & \ddots & \\
& & & \ddots & \ddots & -t_{3} \\
& & & & -t_{1} & -t_{2}
\end{array}\right] .
$$

Using Lemma 2 one verifies that there are no subspaces $U \neq 0, K^{n}$ of $K^{n}$ with $\operatorname{dim}\left(\sum_{i} A_{i} U\right) \leq \operatorname{dim} U$.

Proof [Proof of Theorem 5]. The description of the null-cone is proved in exactly the same way as for symmetric bilinear forms; we do not repeat the argument here. We proceed to prove the inclusions $C_{k}^{(p)} \subseteq C_{q}^{(p)}$ for the following values of the parameters:

1. $n$ arbitrary, $k$ arbitrary, and $p=2$;

2. $n=2 q-1 \geq 3, k=q-1$, and $p$ arbitrary;

3. $n=2 q-1 \geq 5, k=q-2$, and $p \in\{3,4\}$; or

4. $n=2 q-1 \geq 7, k=q-3$, and $p=3$.

These statements are proved as follows: let $\left(\alpha_{1}, \ldots, \alpha_{p}\right) \in C_{k}^{(p)}$ and let $U \subseteq W$ be a pair with $\operatorname{dim} U=k, \operatorname{dim} W=n-k+1$, and $\alpha_{i}(U, W)=0$ for all $i$. Then the $\alpha_{i}$ induce bilinear forms $\bar{\alpha}_{i}$ on the space $W / U$ of dimension $n-2 k+1$, and we find a subspace $U^{\prime}$ of $W / U$ of dimension $d(n-2 k+1, p)$ that is isotropic relative to all $\bar{\alpha}_{i}$. The pre-image of $U^{\prime}$ in $W$ is then a space of dimension $d(n-2 k+1, p)+k$ and isotropic relative to all $\alpha_{i}$. Using Theorem 6 one finds that for the above values of the parameters this value $d(n-2 k+1, p)+k$ is at least $\left\lfloor\frac{n}{2}\right\rfloor+1$, which shows that $\left(\alpha_{1}, \ldots, \alpha_{p}\right) \in C_{q}^{(p)}$. This proves all inclusions above.

Now we prove that there are no other inclusions among the $C_{k}^{(p)}$ for other values of $n, k$, and $p$. Suppose first that $n=2 q$ is even, $p \geq 3$ and $k \in\{1, \ldots, q\}$. Then we find a $p$-tuple in $C_{k}^{(p)}$ not lying in any other $C_{k^{\prime}}^{(p)}$ by a construction similar to the constructions in the proof of Corollary 1: Let $\alpha_{1}, \alpha_{2}, \alpha_{3}$ be forms with matrices $A_{1}, A_{2}, A_{3}$ for which $t_{1} A_{1}+t_{2} A_{2}+t_{3} A_{3}$ equals

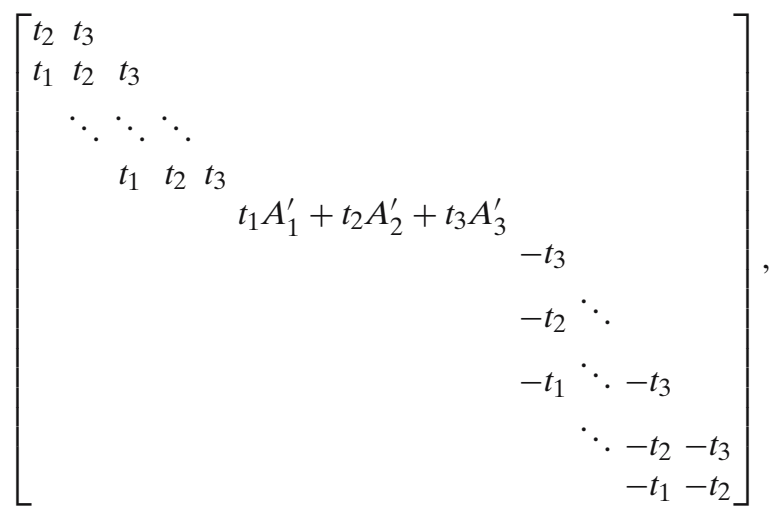


where the diagonal blocks have sizes $(k-1) \times k,(n-2 k+1) \times(n-2 k+1)$, and $k \times(k-1)$ from top left to bottom right, and where the $A_{i}^{\prime}$ are chosen such (skew relative to the skew diagonal) that they map no subspace $U \neq 0, K^{n-2 k+1}$ of $K^{n-2 k+1}$ into a subspace of dimension $<\operatorname{dim} U$; $\operatorname{such} A_{i}^{\prime}$ exist by Corollary 1 . Write $V_{1}:=\left\langle e_{1}, \ldots, e_{k}\right\rangle_{K}$, $V_{2}:=\left\langle e_{k+1}, \ldots, e_{n-k}\right\rangle_{K}$, and $V_{3}:=\left\langle e_{n-k+1}, \ldots, e_{n}\right\rangle_{K}$. Now suppose that $U$ is a subspace of $K^{n}$ for which $\operatorname{dim} \sum A_{i} U<\operatorname{dim} U$. Let $U_{1}:=U \cap V_{1}$, let $U_{2}$ be the projection of $U \cap\left(V_{1} \oplus V_{2}\right)$ to $V_{2}$ along $V_{1}$, and let $U_{3}$ be the projection of $U$ to $V_{3}$ along $V_{1} \oplus V_{2}$. Then $\operatorname{dim} \sum_{i} A_{i} U_{1} \geq \operatorname{dim} U_{1}$ unless $U_{1}=V_{1}, \operatorname{dim} \sum_{i} A_{i} U_{2}>\operatorname{dim} U_{2}$ unless $U_{2}=0$ or $V_{2}$, and $\operatorname{dim} \sum_{i} A_{i} U_{3}>\operatorname{dim} U_{3}$ unless $U_{3}=0$. Summing up these dimensions, we find $\operatorname{dim} \sum_{i} A_{i} U<\operatorname{dim} U$ implies $U_{1}=V_{1}, U_{2}=0$ or $U_{2}=V_{2}$, and $U_{3}=0$. We conclude that $\left(V_{1}, V_{1} \oplus V_{2}\right)$ is the only pair of subspaces $U \subseteq W$ with $\alpha_{i}(U, W)=0$ and $\operatorname{dim} U+\operatorname{dim} W>n$. Hence $\left(\alpha_{1}, \alpha_{2}, \alpha_{3}\right)$ lies in $C_{k}^{(3)}$ but not in any other $C_{k^{\prime}}^{(3)}$.

Next suppose that $n=2 q-1 \geq 9$ is odd. Then we have to show that that $C_{k}^{(3)}$ for $k \notin\{q-1, q-2, q-3\}$ is not contained in any other $C_{k}^{(3)}$. This goes using a construction similar to that above for even $n$, choosing the $A_{i}^{\prime}$-now square skew matrices of size $n-2 k+1=2(q-k) \geq 8-$ such that for all spaces $U$ with $0 \subsetneq U \subsetneq K^{2(q-k)}$ we have $\operatorname{dim} A_{1}^{\prime} U+A_{2}^{\prime} U+A_{3}^{\prime} U>\operatorname{dim} U$; such matrices exist by Corollary 1 .

Next, assuming $n=2 q-1 \geq 7$, suppose that $p \geq 4$ and $k \in\{1, \ldots, q-3, q\}$. By writing down an appropriate standard quadruple of skew matrices $\left(A_{1}, \ldots, A_{4}\right)$ we show that $C_{k}^{(p)}$ is not contained in any other $C_{k^{\prime}}^{(p)}$ : take $A_{1}, A_{2}, A_{3}, A_{4}$ such that $\sum_{i} t_{i} A_{i}$ has the block shape of (11), where the outer two blocks are unchanged (i.e., $A_{4}$ has no non-zero entries there), but the inner block of size $2(q-k) \geq 6$ is as follows:

$$
\left[\begin{array}{cccccccc}
t_{2} & t_{3} & t_{4} & & & & \\
0 & t_{2} & \ddots & \ddots & & & & \\
t_{1} & 0 & \ddots & t_{3} & t_{4} & & & \\
& \ddots & \ddots & t_{2} & 0 & -t_{4} & & \\
& & t_{1} & 0 & -t_{2} & -t_{3} & \ddots & \\
& & & -t_{1} & 0 & \ddots & \ddots & -t_{4} \\
& & & & \ddots & \ddots & -t_{2} & -t_{3} \\
& & & & & -t_{1} & 0 & -t_{2}
\end{array}\right]
$$

Again, applying Lemma 2, one readily verifies that this quadruple of skew matrices does not map any space $U$ into a space of dimension $\leq \operatorname{dim} U$.

A similar construction for $n=2 q-1 \geq 5$ with the following $4 \times 4$-block in the middle:

$$
\left[\begin{array}{cccc}
t_{3} & t_{4} & t_{5} & 0 \\
t_{2} & t_{3} & 0 & -t_{5} \\
t_{1} & 0 & -t_{3} & -t_{4} \\
0 & -t_{1} & -t_{2} & -t_{3}
\end{array}\right]
$$

shows that on $p \geq 5$ copies the set $C_{q-2}^{(p)}$ is not contained in any other $C_{k}^{(p)}$, either.

Finally, we settle the question, for $n$ even, of when the null-cone on $p$ copies of $\bigwedge^{2}\left(V^{*}\right)$ is defined by the polarisations of the Pfaffian. 
Theorem 7. The null-cone $\mathcal{N}\left(\bigwedge^{2}\left(V^{*}\right)^{p}\right)$ with $\operatorname{dim} V=: n$ even is defined by the polarisations of the Pfaffian if and only if either $p=2$ or $n \in\{2,4\}$.

Proof. The proof for $p=2$ goes exactly as for symmetric bilinear forms, and for $n=2$ the statement is trivial.

Suppose therefore that $n=4$. As the referee of this paper kindly pointed out, the theorem for this case can be proved using classical invariant theory: the image of $\mathrm{SL}_{4}$ in $\mathrm{GL}\left(\bigwedge^{2} K^{4}\right)$ is precisely $\mathrm{SO}_{6}$, hence by the First Fundamental Theorem for $\mathrm{SO}_{6}[9]$ one knows precisely the invariants on $p$ copies of this representation, and from this knowledge one can deduce that the null-cone is defined by the polarisations of the invariants on one copy.

In keeping with the more geometrical arguments used for the case of symmetric bilinear forms, we include a short, self-contained proof for the case where $n=4$ that does not rely on classical invariant theory: Let $\mathcal{A}$ be a vector space consisting of singular skew forms on $K^{4}$. We have to show that either the radicals of all forms in $\mathcal{A}$ intersect in a projective point, or there exist a line $U$ and a plane $W \supseteq U$ in $\mathbb{P}^{3}$ with $\alpha(U, W)=0$ for all $\alpha \in A$. By the statement for $p=2$ we know that any pair of elements in $\mathcal{A}$ is of one of these two types.

We prove that in fact every pair $\alpha, \beta \in \mathcal{A}$ is of the first type. Indeed, take $\alpha, \beta \in \mathcal{A}$ non-zero (and hence of $\operatorname{rank} 2$ ), suppose that $\operatorname{rad} \alpha$ and $\operatorname{rad} \beta$ are disjoint lines in $\mathbb{P}^{3}$, and let $U \subseteq W$ be a line and a plane in $\mathbb{P}^{3}$ such that $\alpha(U, W)=\beta(U, W)=0$. For dimension reasons, $U$ must intersect both $\operatorname{rad} \alpha$ and $\operatorname{rad} \beta$, and hence $U$ is distinct from both of these lines. But then the $\alpha$-perp of $U$ and the $\beta$-perp of $U$ are both planes containing $W$, and hence equal to $W$. On the other hand, the radicals of $\alpha$ and $\beta$ are contained in the $\alpha$-perp and the $\beta$-perp of $U$, respectively, hence in $W$. But this contradicts the assumption that the projective lines $\mathbb{P} \operatorname{rad} \alpha$ and $\mathbb{P} \operatorname{rad} \beta$ do not intersect.

We conclude that all radicals of elements in $\mathcal{A}$ intersect. But then they all lie in some plane $W$. Now if $U$ is any line in $W$, then $\alpha(U, W)=0$ for all $\alpha \in \mathcal{A}$, so that $\mathcal{A}$ "lies in" $C_{2}$. This proves the theorem for $n=4$.

Finally, for $n \geq 6$, we have to exhibit a triple of skew bilinear forms that is not nilpotent but whose span lies in the null-cone on $\bigwedge^{2} V^{*}$. Choose for instance $\alpha_{1}, \alpha_{2}, \alpha_{3}$ with matrices $A_{1}, A_{2}, A_{3}$ such that

$$
t_{1} A_{1}+t_{2} A_{2}+t_{3} A_{3}=\left[\begin{array}{ccccccc}
t_{2} & & & & & & \\
& \ddots & & & & & \\
& & & & & & \\
& & & S & & & \\
& & S & 0 & & & \\
& & & -t_{2} & & \\
& & & & \ddots & \\
& & & & & -t_{2}
\end{array}\right], \quad \text { where } S=\left[\begin{array}{cccc}
t_{2} & t_{3} & 0 \\
t_{1} & 0 & -t_{3} \\
0 & -t_{1} & -t_{2}
\end{array}\right] .
$$

Using arguments like those for Lemma 2 one verifies that no subspace of $K^{n}$ is mapped by all $A_{i}$ into a strictly smaller subspace. This concludes the proof of the theorem.

\section{$5 \mathrm{SL}(V)$ on arbitrary bilinear forms}

The invariants of $\operatorname{SL}(V)$ on $\left(V^{*} \otimes V^{*}\right)$ are known [1], but in contrast to the situation for linear maps and symmetric bilinear forms, it is not clear from them that the null-cone 
on one copy of $V^{*} \otimes V^{*}$ is irreducible. The following theorem states that it is, and also describes the components in several copies.

Theorem 8. For $p \geq 2$, the null-cone of $\operatorname{SL}(V)$ on $\left(V^{*} \otimes V^{*}\right)^{p}$ has $q:=\left\lfloor\frac{n+1}{2}\right\rfloor$ irreducible components given by

$$
\begin{aligned}
C_{k}^{(p)}:= & \left\{\left(\alpha_{1}, \ldots, \alpha_{p}\right) \mid \exists U \subseteq W \subseteq V: \operatorname{dim} U=k, \operatorname{dim} W=n-k+1,\right. \\
& \text { and } \left.\alpha_{i}(U, W)=\alpha_{i}(W, U)=0 \text { for all } i=1, \ldots, p\right\}, \quad k=1, \ldots, q .
\end{aligned}
$$

On $p=1$ copy the sets $C_{k}^{(1)}$ form a chain $C_{1}^{(1)} \subseteq \cdots \subseteq C_{q}^{(1)}$, and hence the null-cone equals the irreducible set $C_{q}^{(1)}$.

In the proof of this theorem we use the following lemma.

Lemma 3. Let $\beta$ be a symmetric form and $\gamma$ a skew form on the vector space $V$ of dimension $\geq 2$. Then there exists a $\beta$-isotropic $v_{0} \in V$ for which

$$
\operatorname{dim}\left\{v \in V \mid \beta\left(v_{0}, v\right)=\gamma\left(v_{0}, v\right)=0\right\} \geq \operatorname{dim} V-1
$$

Proof. If the radical of $\gamma$ has dimension $\geq 2$, we may take for $v_{0}$ any $\beta$-isotropic vector in $\operatorname{rad} \gamma$. If $\operatorname{rad} \gamma$ has dimension 1 and is spanned by $v_{1}$, then there are two cases: either $v_{1}$ is $\beta$-isotropic and we may set $v_{0}:=v_{1}$, or $V=K v_{1} \oplus V^{\prime}$, where $V^{\prime}:=v_{1}^{\perp_{\beta}}$. Then $\gamma$ is non-degenerate on $V^{\prime}$ and if we find a $v_{0}$ in $V^{\prime}$ satisfying the conclusion of the lemma for $V^{\prime}$ instead of $V$, it also does the trick for $V$, as $\beta\left(v_{1}, v_{0}\right)=\gamma\left(v_{1}, v_{0}\right)=0$.

Hence the case remains where $\gamma$ is non-degenerate. Let $B, C$ be the linear maps corresponding to $\beta, \gamma$ relative to (.,.) and choose any eigenvector $v_{0}$ of $C^{-1} B$. Then we have $B v_{0} \in K C v_{0}$ so that $\gamma\left(v, v_{0}\right)\left(=\left(v, C v_{0}\right)\right)=0$ implies $\beta\left(v, v_{0}\right)\left(=\left(v, B v_{0}\right)\right)=0$. In particular, $v_{0}$ is $\beta$-isotropic, and the vector space on the left-hand side in the lemma is the $\gamma$-perp of $v_{0}$.

Proof (Proof of Theorem 8). For the first statement, let $\left(\alpha_{1}, \ldots, \alpha_{p}\right)$ be a nilpotent $p$-tuple of bilinear forms and write $\alpha_{i}=\beta_{i}+\gamma_{i}$ for all $i$, with $\beta_{i}$ symmetric and $\gamma_{i}$ skew. Let $B_{i}, C_{i}$ be the linear maps associated $\beta_{i}, \gamma_{i}$, respectively. By assumption there exists a one-parameter subgroup $\lambda: K^{*} \rightarrow \operatorname{SL}(V)$ with $\lim _{t \rightarrow 0} \lambda(t) \alpha_{i}=0$ for all $i$. But this implies that also $\lambda(t) \beta_{i}, \lambda(t) \gamma_{i} \rightarrow 0$ for $t \rightarrow 0$. A fortiori, the $2 p$-tuple $\left(B_{1}, \ldots, B_{p}, C_{1}, \ldots, C_{p}\right)$ is nilpotent under the larger group $\operatorname{SL}(V) \times \operatorname{SL}(V)$, and by Theorem 1 there exist subspaces $U^{\prime}, U^{\prime \prime} \subseteq V$ of dimensions $k$ and $k-1$ such that $B_{i} U^{\prime}, C_{i} U^{\prime} \subseteq U^{\prime \prime}$ for all $i$. Let $W^{\prime}$ be the perp of $U^{\prime}$ relative to our fixed form (.,.), set $U:=U^{\prime} \cap W^{\prime}$ and $W:=W^{\prime}+U^{\prime}$. Then $U \subseteq W, \operatorname{dim} U+\operatorname{dim} W=n+1$, and $\beta_{i}(U, W)=\gamma_{i}(U, W)=0$. But then also $\alpha_{i}(U, W)=\alpha_{i}(W, U)=0$, as claimed.

Now we prove $C_{k}^{(1)} \subseteq C_{k+1}^{(1)}$ for $k<q$. To this end, let $U \subseteq W$ be subspaces of $V$ with $\operatorname{dim} U+\operatorname{dim} W=n+1$. We want to prove that a form $\alpha \in V^{*} \otimes V^{*}$ lying in $C_{k}^{(1)}$ by virtue of $\alpha(U, W)=\alpha(W, U)=0$ also lies in $C_{k+1}^{(1)}$. Indeed, write $\alpha=\beta+\gamma$, where $\beta$ is symmetric and $\gamma$ is skew. The forms $\beta, \gamma$ induce a symmetric form $\bar{\beta}$ and a skew-symmetric form $\bar{\gamma}$ on $W / U$, respectively, and by the preceding lemma there exists a $\bar{w}_{0} \in W / U$ for which

$$
\operatorname{dim}\left\{\bar{w} \in W / U \mid \bar{\beta}\left(\bar{w}, \bar{w}_{0}\right)=\bar{\gamma}\left(\bar{w}, \bar{w}_{0}\right)=0\right\} \geq \operatorname{dim} W / U-1 .
$$

Let $w_{0}$ be a pre-image of $\bar{w}$ in $W$, set $U^{\prime}:=U \oplus K w_{0}$, and let $W^{\prime} \subseteq W$ be a subspace of codimension 1 that contains $w_{0}$ and whose image in $W / U$ is contained in the 
space above. Then we still have $\alpha\left(U^{\prime}, W^{\prime}\right)=0$ and $\operatorname{dim} U^{\prime}+\operatorname{dim} W^{\prime}=n+1$, but now $\operatorname{dim} U^{\prime}=k+1$, as claimed.

Finally, we have to show that on $p \geq 2$ copies there are no inclusions among the sets $C^{(k)}$ with $k=1, \ldots, q$ are distinct. But their intersections with the set of $p$-tuples of symmetric bilinear forms are already distinct, see Theorem 3.

The last question to be answered here is whether the polarisations of the invariants on one copy of $V^{*} \otimes V^{*}$ define the null-cone on more copies. The answer can be deduced from the answers for symmetric forms and for skew forms.

Theorem 9. The null-cone of $\operatorname{SL}(V)$ on $\left(V^{*} \otimes V^{*}\right)^{p}$ is defined by the polarisations to $p \geq 2$ copies of the invariants on $V^{*} \otimes V^{*}$ if and only if $\operatorname{dim} V \leq 2$.

Proof. For $\operatorname{dim} V=1$ the statement is trivial. Suppose that $\operatorname{dim} V=2$ and let $\mathcal{A}$ be a space of nilpotent bilinear forms on $V$. If $\alpha \in \mathcal{A}$, then by Theorem 8 both the symmetric component and the skew component of $\alpha$ are singular. As the skew component has even rank, it is then zero. Hence $\mathcal{A}$ consists of symmetric forms only, and therefore the existence of a common radical for forms in $\mathcal{A}$ follows from Theorem 4 .

Suppose now that $n \geq 3$. Let $\beta_{1}, \beta_{2}, \gamma_{1}$ be the bilinear forms on $K^{n}$ whose matrices $B_{1}, B_{2}, C_{1}$ relative to the orthogonal sum (.,.) of the skew diagonal forms on $K^{3}$ and $K^{n-3}$ satisfy

$$
s_{1} B_{1}+s_{2} B_{2}+t_{1} C_{1}=\left[\begin{array}{ccc|c}
s_{1} & s_{2} & 0 & \\
t_{1} & 0 & s_{2} & \\
0 & -t_{1} & s_{1} & \\
\hline & & & s I_{n-3}
\end{array}\right] .
$$

A direct computation shows that $\operatorname{det}\left(s_{1} B_{1}+s_{2} B_{2}+t_{1} C_{1}\right)$ is identically zero. We claim that actually $\mathcal{A}:=\left\langle\beta_{1}, \beta_{2}, \gamma_{1}\right\rangle_{K}$ consists entirely of nilpotent bilinear forms; as the determinant is not the only invariant, the preceding computation does not prove this yet. But let $\alpha$ be in $\mathcal{A}$ with matrix $A$. Then $A^{t}$-where transposition, as always, is relative to the form (., .) - defines the form $\alpha^{t}$, which by the definition of $\mathcal{A}$ also lies in $\mathcal{A}$ and the singular matrix pencil $\left\langle A, A^{t}\right\rangle_{K}$ has a subspace $U$ of $K^{n}$ for which $W^{\prime}:=A^{t} U+A U$ has dimension $<\operatorname{dim} U$. But then the perp $W$ of $W^{\prime}$ relative to (., .) is a subspace of $K^{n}$ of dimension $>n-\operatorname{dim} U$ satisfying $\alpha(W, U)=\alpha^{t}(W, U)(=\alpha(U, W))=0$. Replacing $(U, W)$ by the pair $(U \cap W, U+W)$ as usual, we find a witness for the nilpotency of $\alpha$.

However, the pair $\left(\beta_{1}+\gamma_{1}, \beta_{2}\right)$ of bilinear forms is not nilpotent. Indeed, if it were, then there would be $U \subseteq W$ with $\operatorname{dim} U+\operatorname{dim} W=n+1$ and $\beta_{1}(U, W)=\beta_{2}(U, W)=$ $\gamma_{1}(U, W)=0$, i.e., with $\operatorname{dim} B_{1} U+B_{2} U+C_{1} U<\operatorname{dim} U$. By Lemma 2 no $U$ with this property exists.

\section{References}

1. Adamovich, O., Golovina, E.: Invariants of a pair of bilinear forms. Mosc. Univ. Math. Bull. 32(2), 11-14 (1977)

2. Bollobás, B.: Modern graph theory, Graduate Texts in Mathematics, vol. 184. Springer, Berlin Heidelberg New York (1998)

3. Buhler, J., Gupta, R., Harris, J.: Isotropic subspaces of skewforms and maximal abelian subgroups of p-groups. J. Algebra 108, 269-279 (1987)

4. Draisma, J.: Counting components of the null-cone on tuples. Preprint (2005). Presented at MEGA 2005, to appear in Transformation Groups.

5. Draisma, J.: Small maximal spaces of non-invertible matrices. Bull. Lond. Math. Soc. (Preprint, 2005, to appear)

盽 Springer 
6. Draisma, J., Kemper, G., Wehlau, D.: Polarization of separating invariants (Submitted, 2005)

7. Eisenbud, D., Harris, J.: Vector spaces of matrices of low rank. Adv. Math. 70(2), 135-155 (1988)

8. Gantmacher, F.: The theory of matrices, vol. 2. AMS Chelsea Publishing, New York (1959)

9. Goodman, R., Wallach, N.R.: Representations and Invariants of the Classical Groups. Cambridge University Press, Cambridge (1998)

10. Harris, J.: Algebraic geometry. A first course. No. 133 in Graduate Texts in Mathematics. Springer, Berlin Heidelberg New York (1992)

11. Hesselink, W.H.: Desingularizations of varieties of nullforms. Invent. Math. 55, 141-163 (1979)

12. Knop, F.: On Noethers and Weyl's bound in positive characteristic. In: Invariant theory in all Characteristics. CRM Proceedings of Lecture Notes, vol. 35, pp. 175-188. Am. Math. Soc., Providence, RI (2004)

13. Kraft, H.: Geometrische Methoden in der Invariantentheorie. Friedr. Vieweg \& Sohn, Braunschweig/Wiesbaden (1984)

14. Kraft, H., Wallach, N.R.: On the nullcone of representations of reductive groups. Pac. J. Math. (Preprint, 2005).

15. Mumford, D., Fogarty, J., Kirwan, F.: Geometric invariant theory, Ergebnisse der Mathematik und ihrer Grenzgebiete, 3. Folge, vol. 34. Springer, Berlin Heidelberg New York (1993)

16. Popov, V., Vinberg, E.: Invariant Theory, Encyclopaedia of Mathematical Sciences, vol. 55, chap. II. Springer, Berlin Heidelberg New York (1994)

17. Wall, C.: Nets of quadrics, and theta-characteristics of singular curves. Philos. Trans. Roy. Soc. London Ser. A 289(1357), 229-269 (1978)

18. Weyl, H.: The classical groups, their invariants and representations. Princeton University Press, Princeton (1939) 